Supporting information for:

\title{
Physicochemical Profiling and Comparison of Research Antiplasmodials and Advanced Stage Antimalarials with Oral Drugs \\ Amritansh Bhanot ${ }^{\ddagger}$, Sandeep Sundriyal ${ }^{{ }^{*}}$
}

${ }^{\dagger}$ Department of Pharmacy, Birla Institute of Technology and Science-Pilani (BITS), Pilani

Campus, Vidya Vihar, Pilani, Rajasthan 333 031, India.

*Email: sandeep.sundriyal@pilani.bits-pilani.ac.in 
Table S1: Details of ASAM category of molecules

\begin{tabular}{|c|c|c|c|c|}
\hline S.No & Drug & Structure & Status & Reference \\
\hline 1 & Amadoquine & & Marketed & \\
\hline 2 & Arteether & & Marketed & \\
\hline 3 & Artemether & & Marketed & \\
\hline 4 & Artemisinin & & Marketed & \\
\hline 5 & Artesunate & & Marketed & \\
\hline 6 & Atovaquone & & Marketed & \\
\hline 7 & Azithromycin & & Marketed & \\
\hline 8 & Bulaquine & & Marketed & \\
\hline 9 & Chloroquine & & Marketed & \\
\hline 10 & Chlorproguanil & & Marketed & \\
\hline 11 & Clindamycin & & Marketed & \\
\hline 12 & Cycloguanil & & Marketed & \\
\hline 13 & Dapsone & & Marketed & \\
\hline 14 & Dihydroartemisinin & & Marketed & \\
\hline 15 & Doxycycline & & Marketed & \\
\hline 16 & Halofantrine & & Marketed & \\
\hline 17 & Lumefantrine & & Marketed & \\
\hline 18 & Mefloquine & & Marketed & \\
\hline 19 & Naphthoquine & & Marketed & \\
\hline 20 & $\begin{array}{l}\text { N- } \\
\text { Desethylamodiaquin } \\
\text { e }\end{array}$ & & Marketed & \\
\hline 21 & OZ277/Arterolane & & Marketed & \\
\hline 22 & Piperaquine & & Marketed & \\
\hline 23 & Primaquine & & Marketed & \\
\hline 24 & Proguanil & & Marketed & \\
\hline 25 & Pyrimethamine & & Marketed & \\
\hline 26 & Pyronaridine & & Marketed & \\
\hline 27 & Quinidine & & Marketed & \\
\hline 28 & Quinine & & Marketed & \\
\hline 29 & Sulfadoxine & & Marketed & \\
\hline 30 & Sulfalene & & Marketed & \\
\hline 31 & Sulfamethoxazole & & Marketed & \\
\hline 32 & Tafenoquine & & Marketed & \\
\hline 33 & Tetracycline & & Marketed & \\
\hline 34 & AQ 13 & & Phase 2 & $\begin{array}{c}\text { Koita OA, Sangaré L, } \\
\text { Miller HD, Sissako A, } \\
\text { Coulibaly M, Thompson } \\
\text { TA, et al. AQ-13, an } \\
\text { investigational } \\
\text { antimalarial, versus } \\
\text { artemether plus } \\
\text { lumefantrine for the } \\
\text { treatment of } \\
\text { uncomplicated }\end{array}$ \\
\hline
\end{tabular}




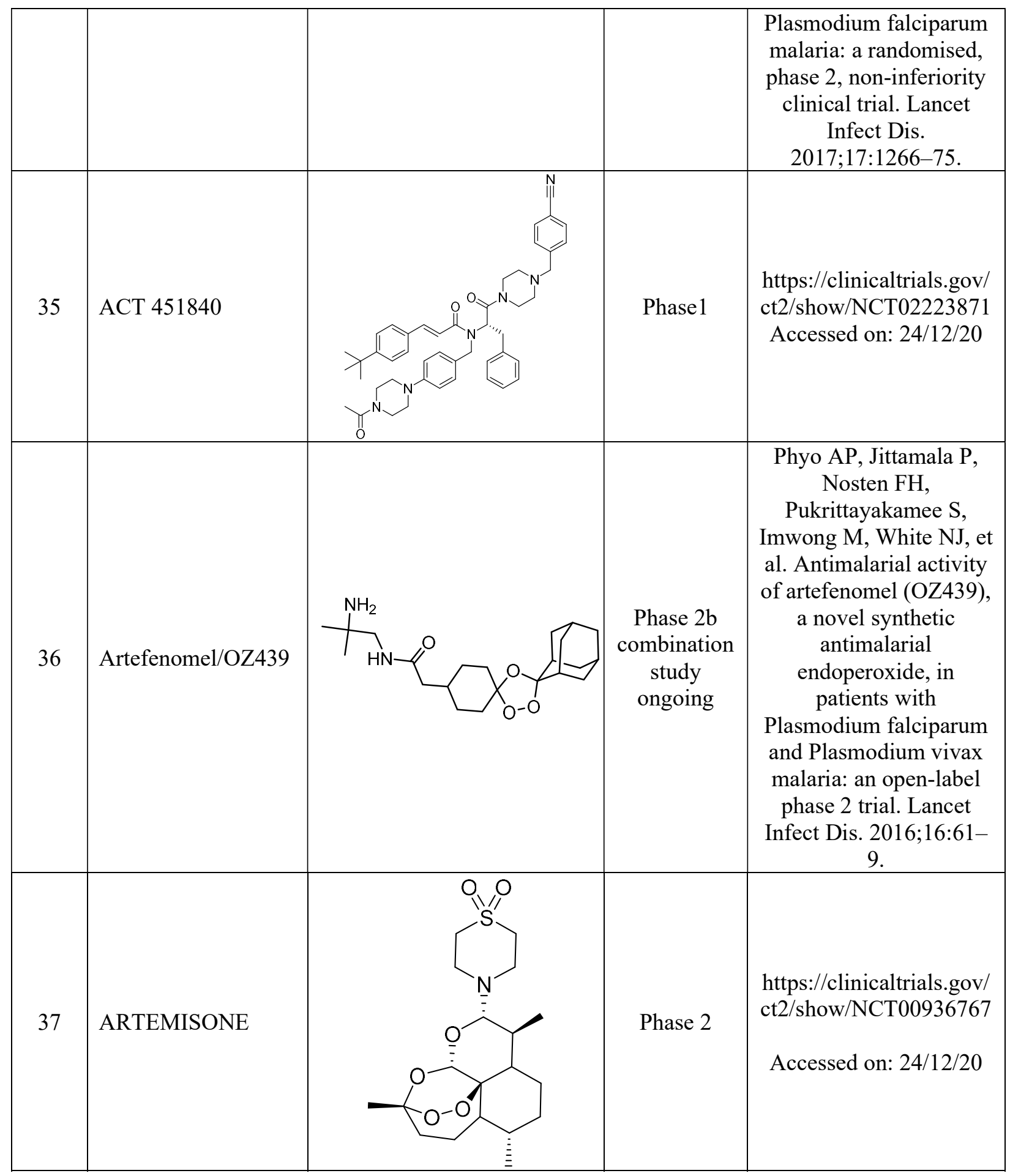




38 CDRI 9778 39 (2)




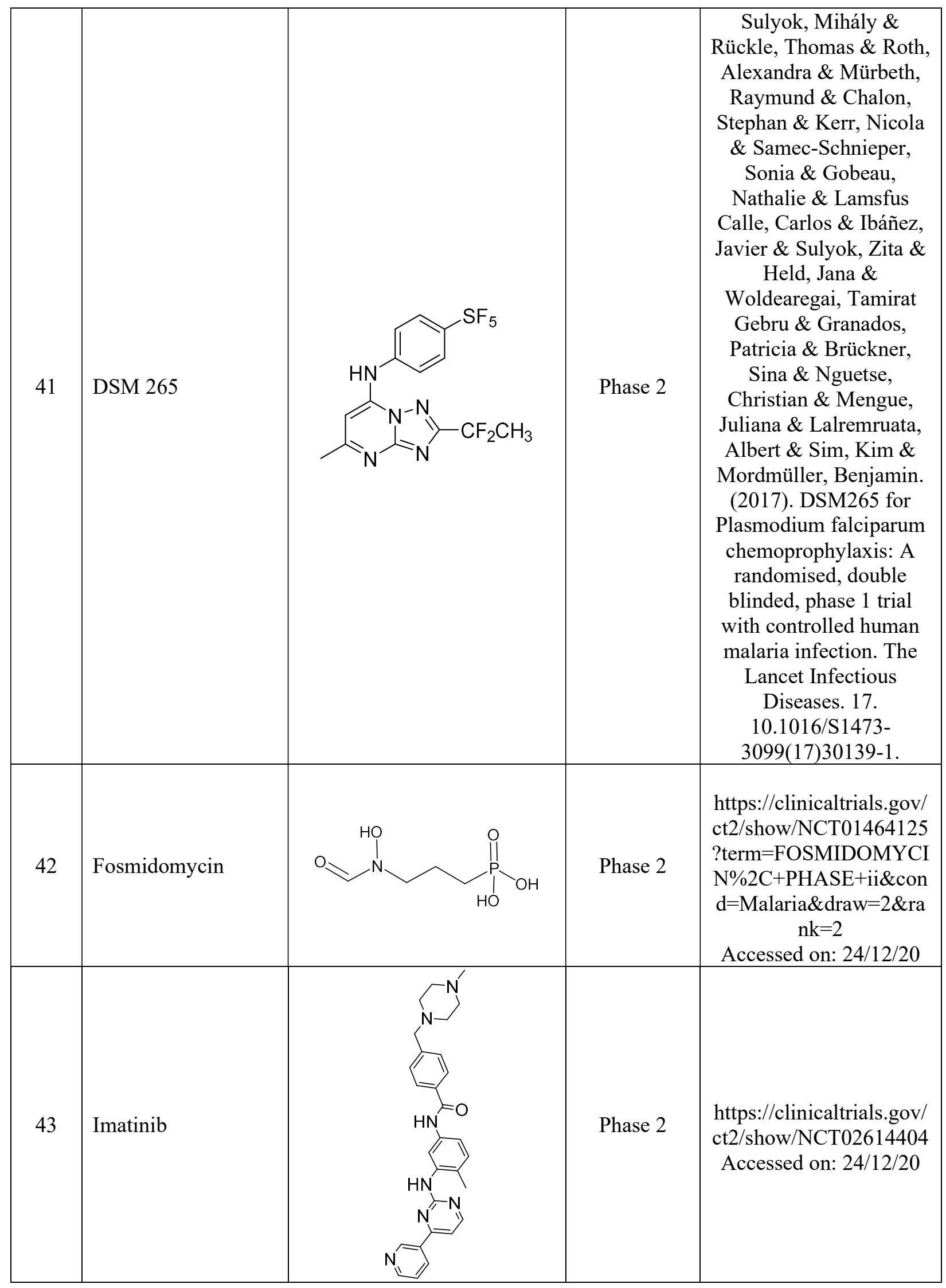




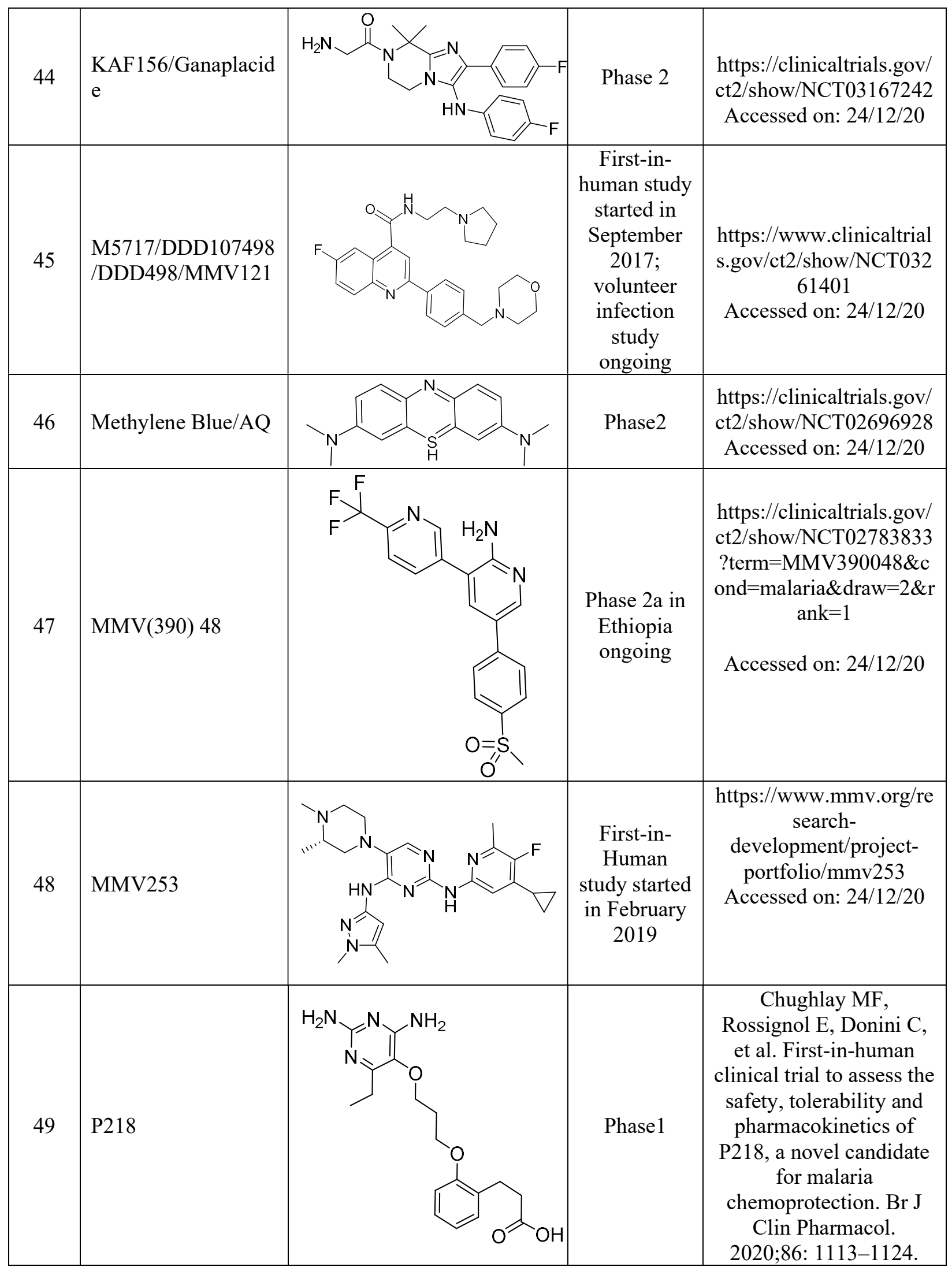




\begin{tabular}{|c|c|c|c|c|}
\hline & & & & $\begin{array}{c}\text { https://doi.org/10.1111/b } \\
\text { cp.14219 }\end{array}$ \\
\hline 50 & Rosiglitazone & & Phase $2 \mathrm{a}$ & $\begin{array}{l}\text { Rosauro Varo, Valerie } \\
\text { M. Crowley, Antonio } \\
\text { Sitoe, Lola Madrid, } \\
\text { Lena Serghides, Rubao } \\
\text { Bila, Helio Mucavele, } \\
\text { Alfredo Mayor, Quique } \\
\text { Bassat \& Kevin C. Kain. } \\
\text { Safety and tolerability of } \\
\text { adjunctive rosiglitazone } \\
\text { treatment for children } \\
\text { with uncomplicated } \\
\text { malaria. } 215(2017)\end{array}$ \\
\hline 51 & $\begin{array}{l}\text { SAR97276/Albitiaz } \\
\text { olium bromide }\end{array}$ & $\mathrm{OH}_{\mathrm{H}}$ & Phase2 & $\begin{array}{c}\text { Held J, Supan C, Salazar } \\
\text { CLO, Tinto H, Bonkian } \\
\text { LN, Nahum A, Sié A, } \\
\text { Abdulla S, Cantalloube } \\
\text { C, Djeriou E, Bouyou- } \\
\text { Akotet M, Ogutu B, } \\
\text { Mordmüller B, } \\
\text { Kreidenweiss A, Siribie } \\
\text { M, Sirima SB, Kremsner } \\
\text { PG. Safety and efficacy } \\
\text { of the choline analogue } \\
\text { SAR97276 for malaria } \\
\text { treatment: results of two } \\
\text { phase 2, open-label, } \\
\text { multicenter trials in } \\
\text { African patients. Malar } \\
\text { J. 2017 May } \\
\text { 4;16(1):188. doi: } \\
\text { 10.1186/s12936-017- } \\
\text { 1832-x. }\end{array}$ \\
\hline 52 & SJ733 & $\mathrm{CN}$ & Phase 1 & $\begin{array}{c}\text { Gaur AH, McCarthy JS, } \\
\text { Panetta JC, Dallas RH, } \\
\text { Woodford J, Tang L, } \\
\text { Smith AM, Stewart TB, } \\
\text { Branum KC, Freeman } \\
\text { BB 3rd, Patel ND, John } \\
\text { E, Chalon S, Ost S, } \\
\text { Heine RN, Richardson } \\
\text { JL, Christensen R, Flynn } \\
\text { PM, Van Gessel Y, } \\
\text { Mitasev B, Möhrle JJ, } \\
\text { Gusovsky F, Bebrevska } \\
\text { L, Guy RK. Safety, } \\
\text { tolerability, } \\
\text { pharmacokinetics, and } \\
\text { antimalarial efficacy of a } \\
\text { novel Plasmodium }\end{array}$ \\
\hline
\end{tabular}




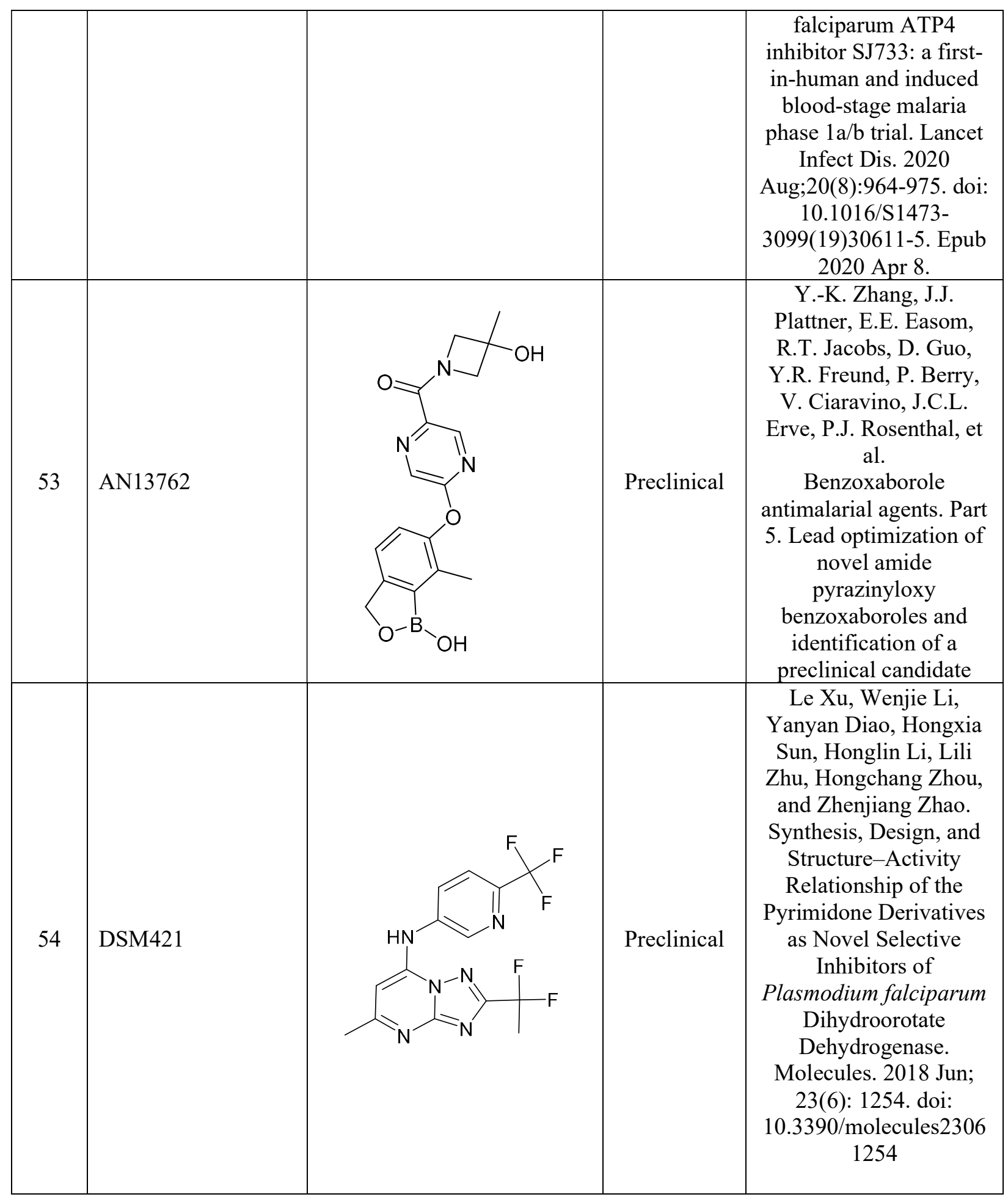




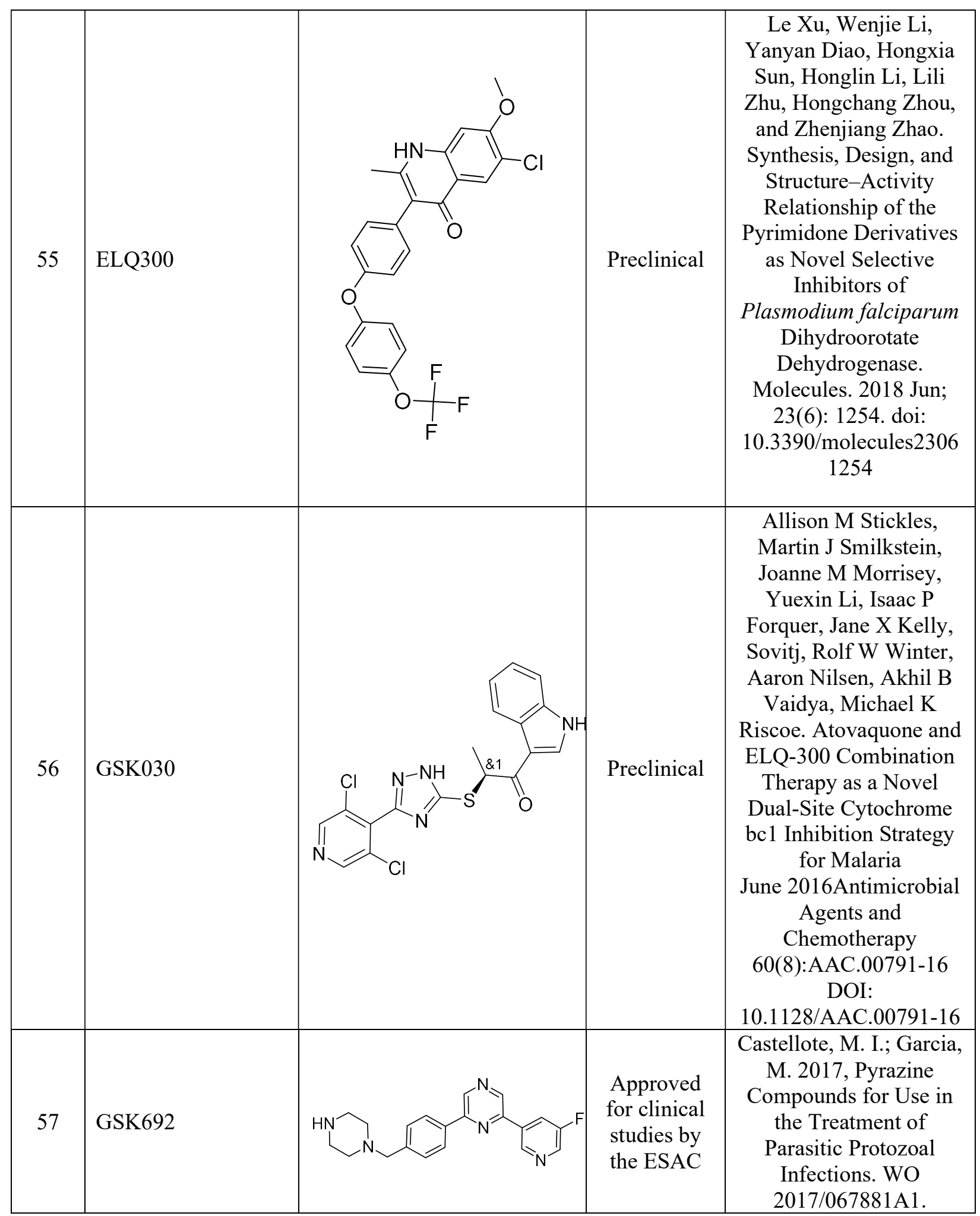




\begin{tabular}{|c|c|c|c|c|}
\hline 58 & JPC3210/MMV646 & & Preclinical & $\begin{array}{c}\text { Birrell GW, Challis MP, } \\
\text { De Paoli A, Anderson } \\
\text { D, Devine SM, } \\
\text { Heffernan GD, Jacobus } \\
\text { DP, Edstein MD, } \\
\text { Siddiqui G, Creek DJ: } \\
\text { Multi-omic } \\
\text { characterisation of the } \\
\text { mode of action of a } \\
\text { potent new antimalarial } \\
\text { compound, JPC-3210, } \\
\text { against Plasmodium } \\
\text { falciparum. Mol Cell } \\
\text { Proteomics. 2019 Dec } \\
\text { 13. pii: RA119.001797. } \\
\text { doi: } \\
\text { 10.1074/mcp.RA119.00 } \\
\text { 1797. }\end{array}$ \\
\hline 59 & KAI407 & ${ }_{F} \lambda$ & Lead & $\begin{array}{l}\text { Anne-Marie Zeeman, } \\
\text { Sandra M. van } \\
\text { Amsterdam, Case W. } \\
\text { McNamara, Annemarie } \\
\text { Voorberg-van der Wel, } \\
\text { Els J. Klooster, } \\
\text { Alexander van den Berg, } \\
\text { Edmond J. Remarque, } \\
\text { David M. Plouffe, } \\
\text { Geert-Jan van Gemert, } \\
\text { Adrian Luty, Robert } \\
\text { Sauerwein, Kerstin } \\
\text { Gagaring, Rachel } \\
\text { Borboa, Zhong Chen, } \\
\text { Kelli Kuhen, Richard J. } \\
\text { Glynne, Arnab K. } \\
\text { Chatterjee, Advait } \\
\text { Nagle, Jason Roland, } \\
\text { Elizabeth A. Winzeler, } \\
\text { Didier Leroy, Brice } \\
\text { Campo, Thierry T. } \\
\text { Diagana, Bryan K. S. } \\
\text { Yeung, Alan W. } \\
\text { Thomas, Clemens H. M. } \\
\text { Kocken. KAI407, a } \\
\text { Potent Non-8- } \\
\text { Aminoquinoline } \\
\text { Compound That } \\
\text { Kills Plasmodium } \\
\text { cynomolgi Early Dormant } \\
\text { Liver Stage Parasites In } \\
\text { Vitro. Antimicrobial } \\
\text { Agents and }\end{array}$ \\
\hline
\end{tabular}




\begin{tabular}{|c|c|c|c|c|}
\hline & & & & $\begin{array}{c}\text { Chemotherapy. 2014 Mar; } \\
\text { 58(3): 1586-1595 }\end{array}$ \\
\hline 60 & KDU691 & $>_{\mathrm{O}}$ & Preclinical & $\begin{array}{c}\text { Anne-Marie Zeeman,a } \\
\text { Suresh B. } \\
\text { Lakshminarayana,b } \\
\text { Nicole van der Werff,a } \\
\text { Els J. Klooster,a } \\
\text { Annemarie Voorberg- } \\
\text { van der Wel,a Ravinder } \\
\text { R. Kondreddi,b } \\
\text { Christophe } \\
\text { Bodenreider,b Oliver } \\
\text { Simon,b Robert } \\
\text { Sauerwein,c Bryan K. S. } \\
\text { Yeung,b Thierry T. } \\
\text { Diagana,corresponding } \\
\text { authorb and Clemens H. } \\
\text { M. Kocken. PI4 Kinase } \\
\text { Is a Prophylactic but Not } \\
\text { Radical Curative Target } \\
\text { in Plasmodium vivax- } \\
\text { Type Malaria Parasites. } \\
\text { Antimicrob Agents } \\
\text { Chemother. 2016 May; } \\
\text { 60(5): 2858-2863. } \\
\text { doi: } \\
\text { 10.1128/AAC.03080-15 }\end{array}$ \\
\hline 61 & MK 4815 & & Preclinical & $\begin{array}{l}\text { Mary Ann Powles, John } \\
\text { Allocco, Lai Yeung, } \\
\text { Bakela Nare, Paul } \\
\text { Liberator, and Dennis } \\
\text { Schmatz. MK-4815, a } \\
\text { Potential New Oral } \\
\text { Agent for Treatment of } \\
\text { Malaria. Antimicrob } \\
\text { Agents Chemother. 2012 } \\
\text { May; 56(5): 2414-2419. } \\
\text { doi: } \\
\text { 10.1128/AAC.05326-11 }\end{array}$ \\
\hline 62 & MMV183/SSJ-183 & & Preclinical & $\begin{array}{c}\text { https://www.mmv.org/p } \\
\text { ortfolio-type/preclinical. } \\
\text { Accessed on 23/12/20. }\end{array}$ \\
\hline
\end{tabular}




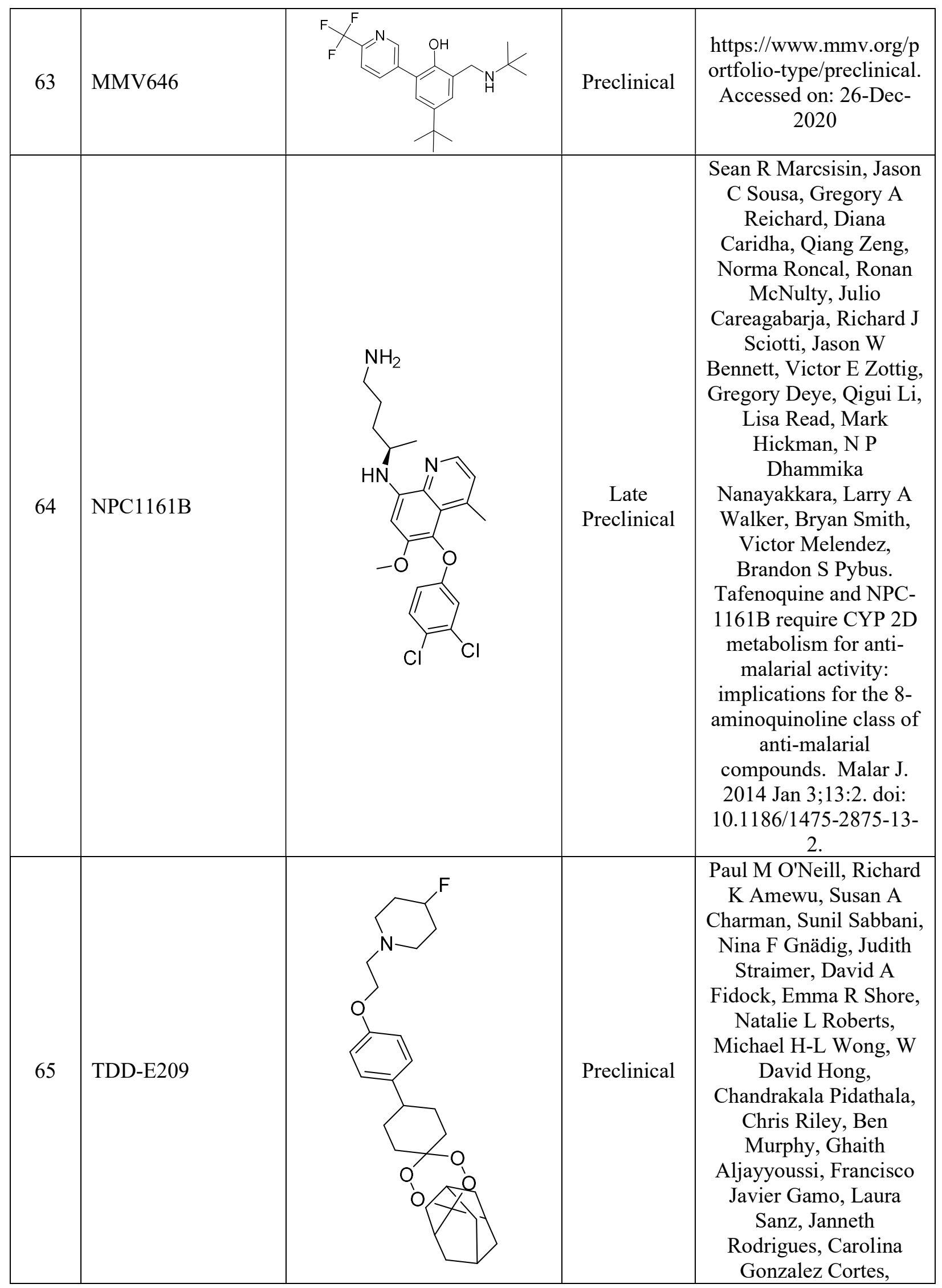




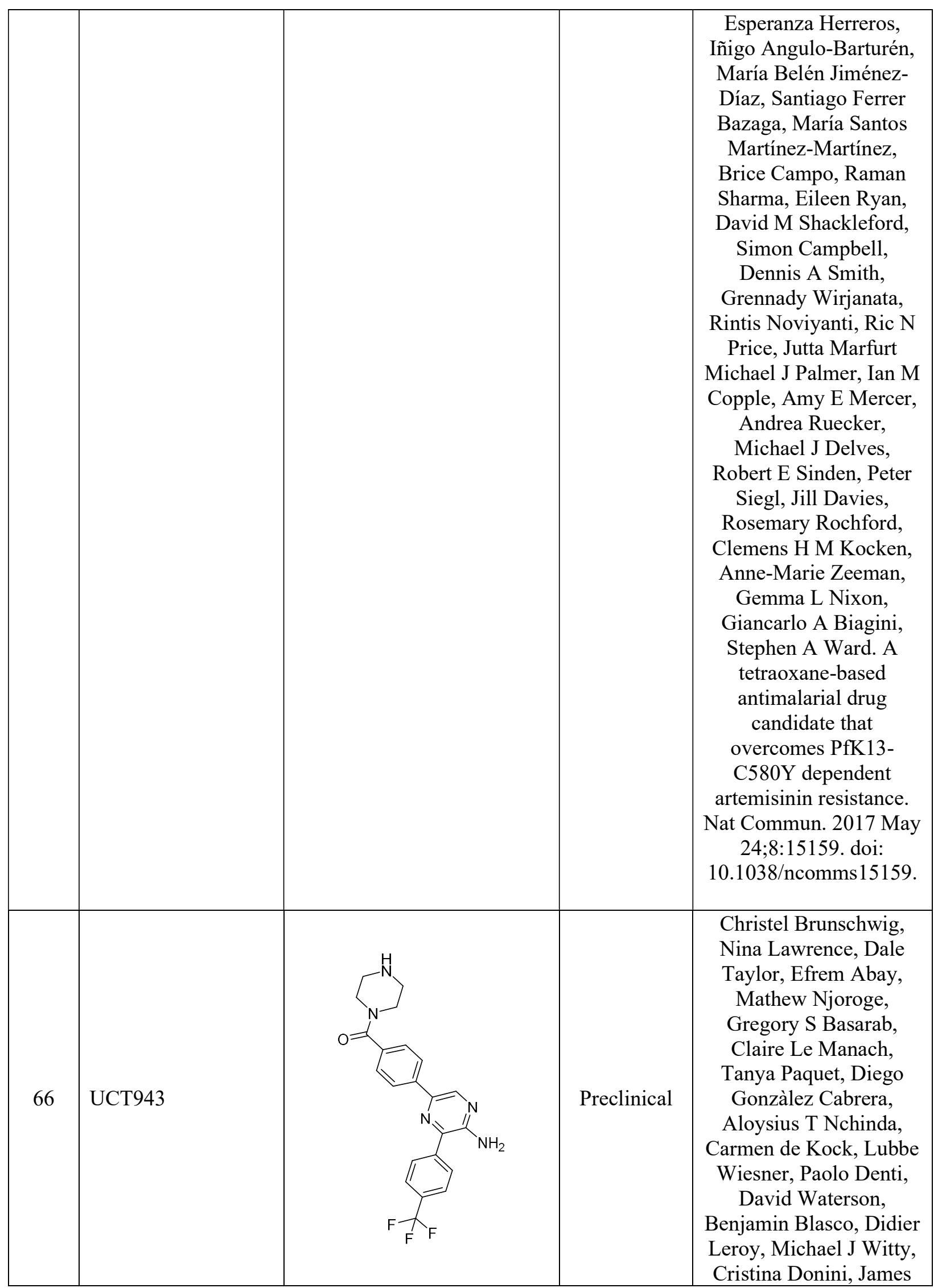




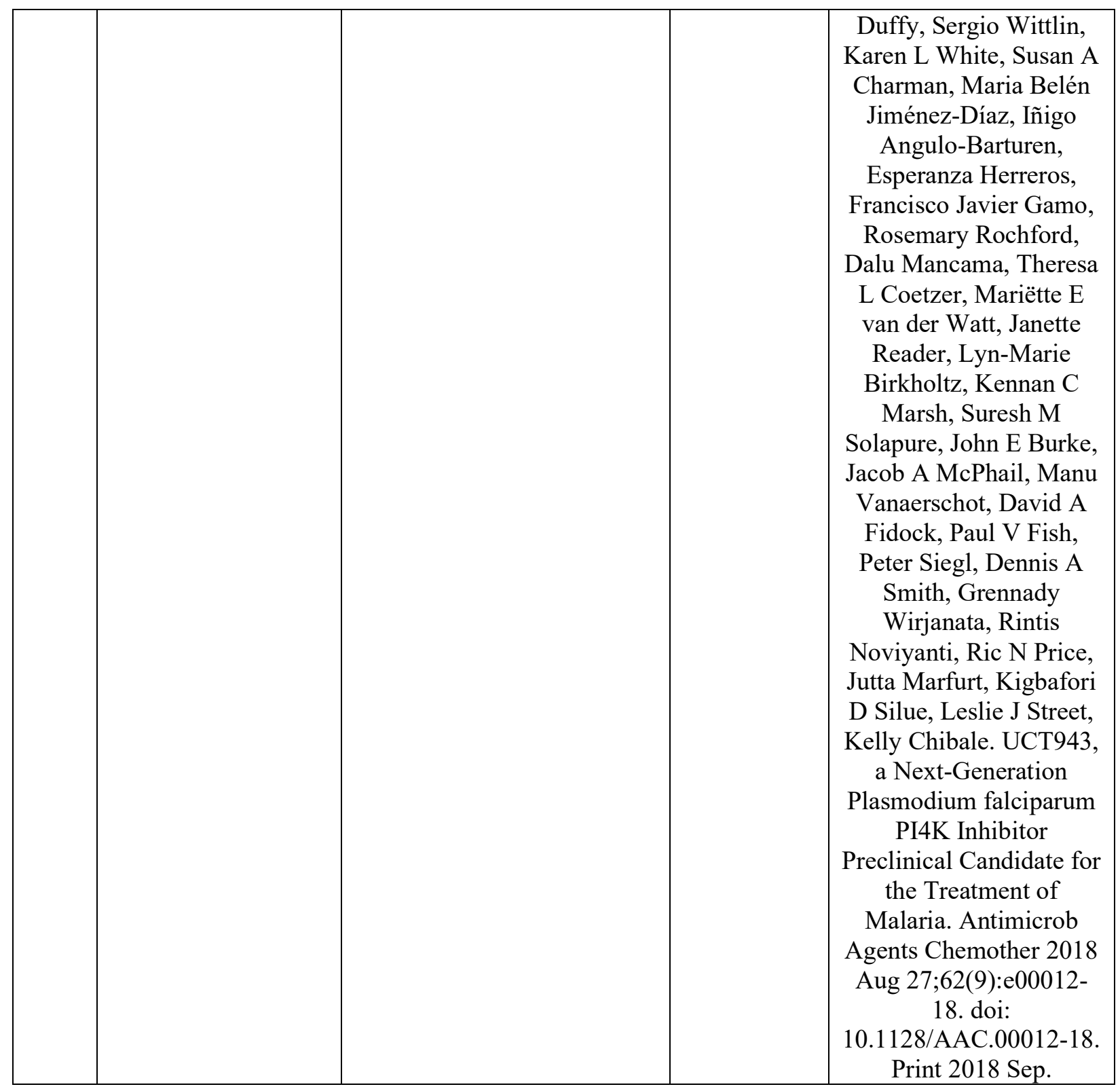


Table S2: Distribution of low $(\mathrm{MW}<500)$ and high $(\mathrm{MW}>500 \mathrm{Da})$ molecular weight compounds in each category

\begin{tabular}{|l|l|l|l|}
\hline Category & Low $(\mathrm{MW}<500 \mathrm{Da})$ & Percentage Low & High $(\mathrm{MW}>500 \mathrm{Da})$ \\
\hline IN & 6037 & $\sim 82 \%$ & 1328 \\
\hline MA & 5432 & $\sim 82 \%$ & 1188 \\
\hline HA & 8036 & $\sim 76 \%$ & 2521 \\
\hline ASAM & 58 & $\sim 88 \%$ & 8 \\
\hline Oral drugs & 1730 & $\sim 88 \%$ & 224 \\
\hline Total & $\mathbf{2 1 2 9 3}$ & $\sim \mathbf{8 0} \%$ & $\mathbf{5 2 6 9}$ \\
\hline
\end{tabular}

Table S3: Distribution of aromatic and aliphatic N-heterocycles in each category.

\begin{tabular}{|l|l|l|l|l|l|}
\hline Category & $\begin{array}{l}\text { Total } \\
\text { molecules }\end{array}$ & $\begin{array}{l}\text { Molecules having } \\
\text { at least } 1 \text { aromatic } \\
\text { N-heterocycle }\end{array}$ & \%age & $\begin{array}{l}\text { Molecules having } \\
\text { at least 1 aliphatic } \\
\text { N-heterocycle }\end{array}$ & \%age \\
\hline IN & 7365 & 2636 & 36 & 2543 & 35 \\
\hline MA & 6620 & 3211 & 49 & 2836 & 43 \\
\hline HA & 10557 & 5978 & 57 & 4771 & 45 \\
\hline ASAM & 66 & 41 & 62 & 25 & 38 \\
\hline $\begin{array}{l}\text { Oral } \\
\text { Drugs }\end{array}$ & 1954 & 591 & 30 & 899 & 46 \\
\hline
\end{tabular}


Table S4: Six most frequently occurring rings in each category

Category


Figure S1: Correlation between Actelion clogP (X-axis) vs the experimental $\log \mathrm{P}$ for a set of 452 drugs reported in the supporting information of reference 4.

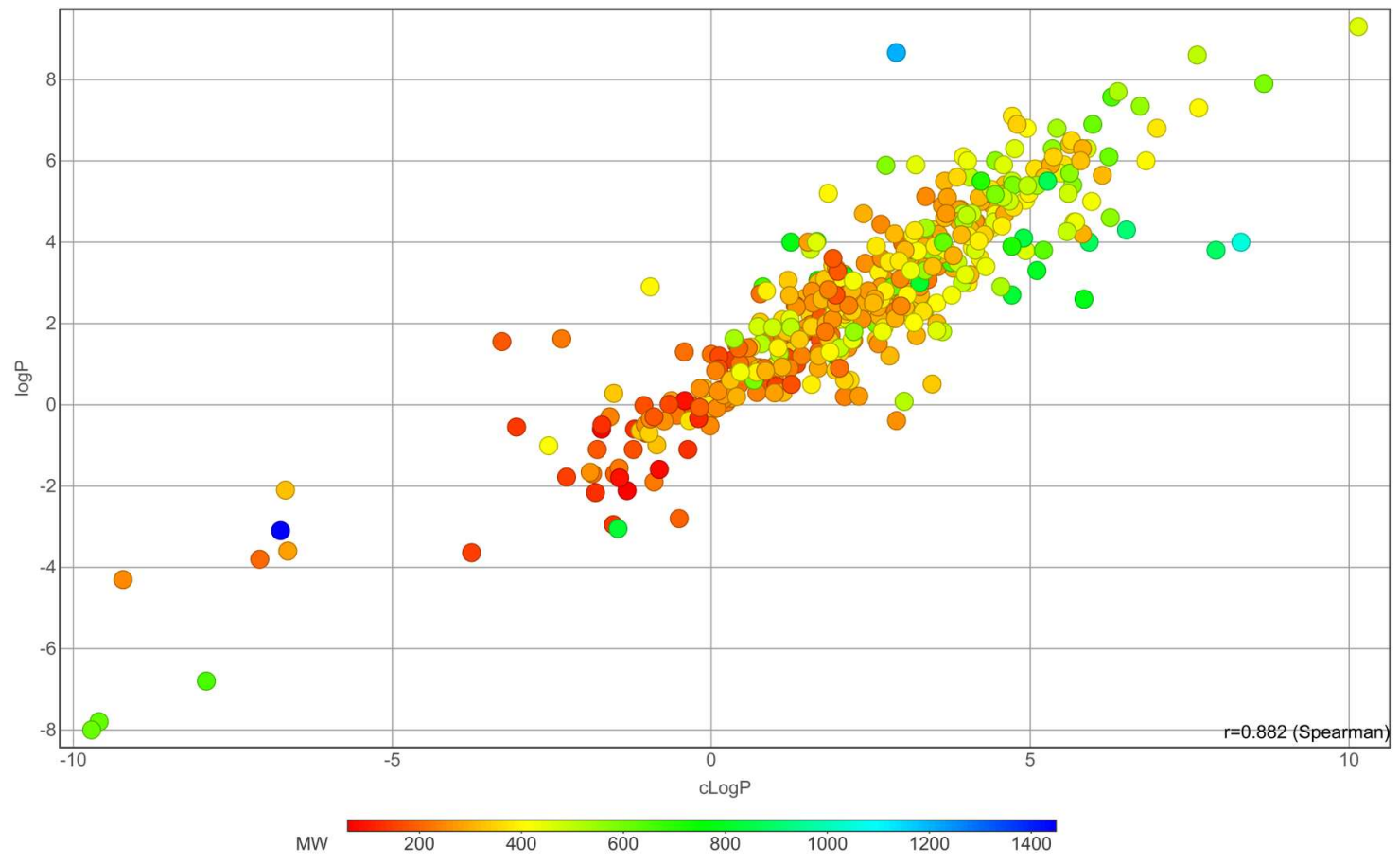

Figure S2: Correlation between Actelion clogP (X-axis) vs the Stardrop clogP for a set of 452 drugs reported in the supporting information of reference 4 .

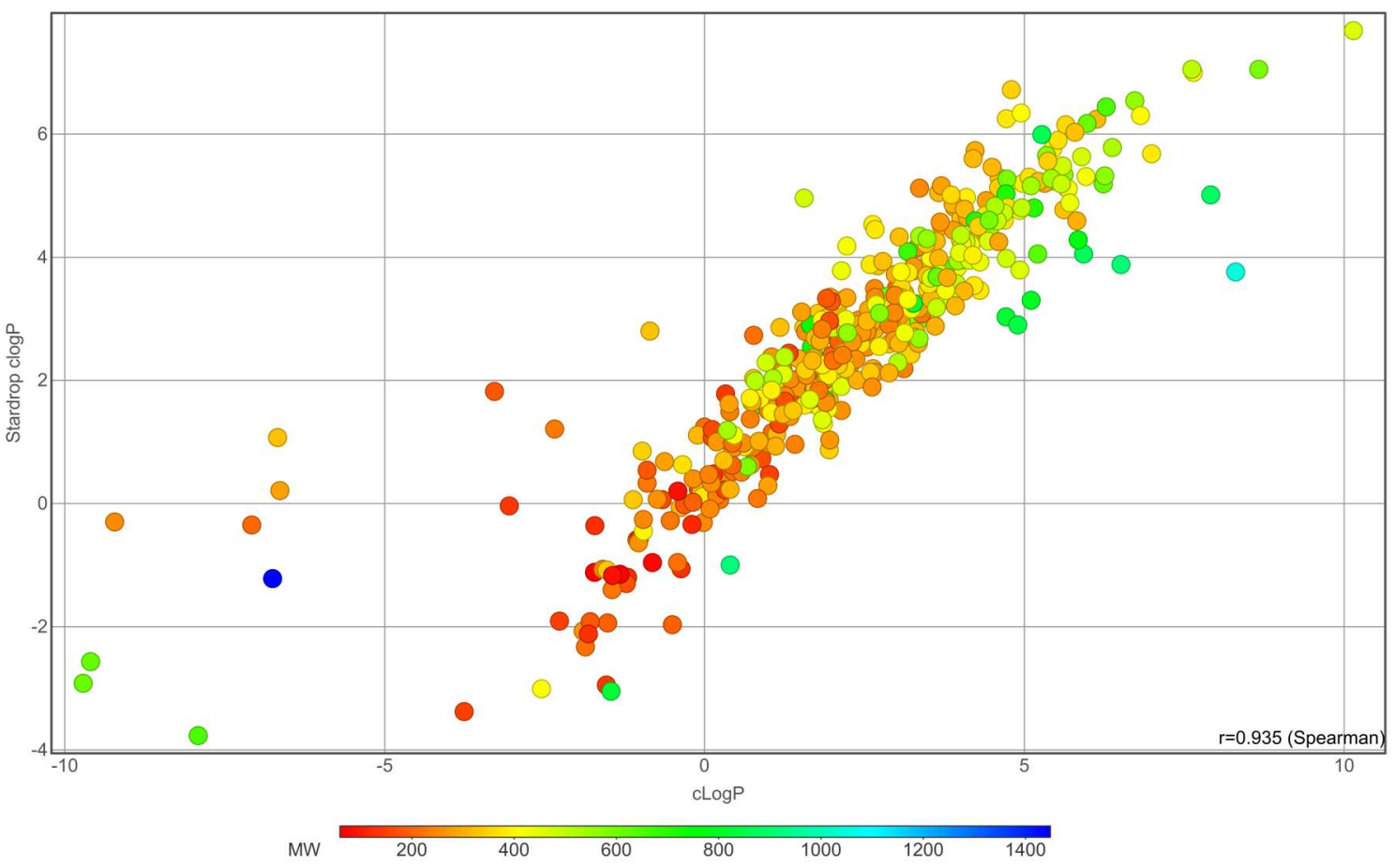


Figure S3: Distribution of mean of clogP among low (MW < 500) and high (MW > 500 Da) molecular weight compounds within the IN, MA, HA, ASAM, and oral drugs categories.

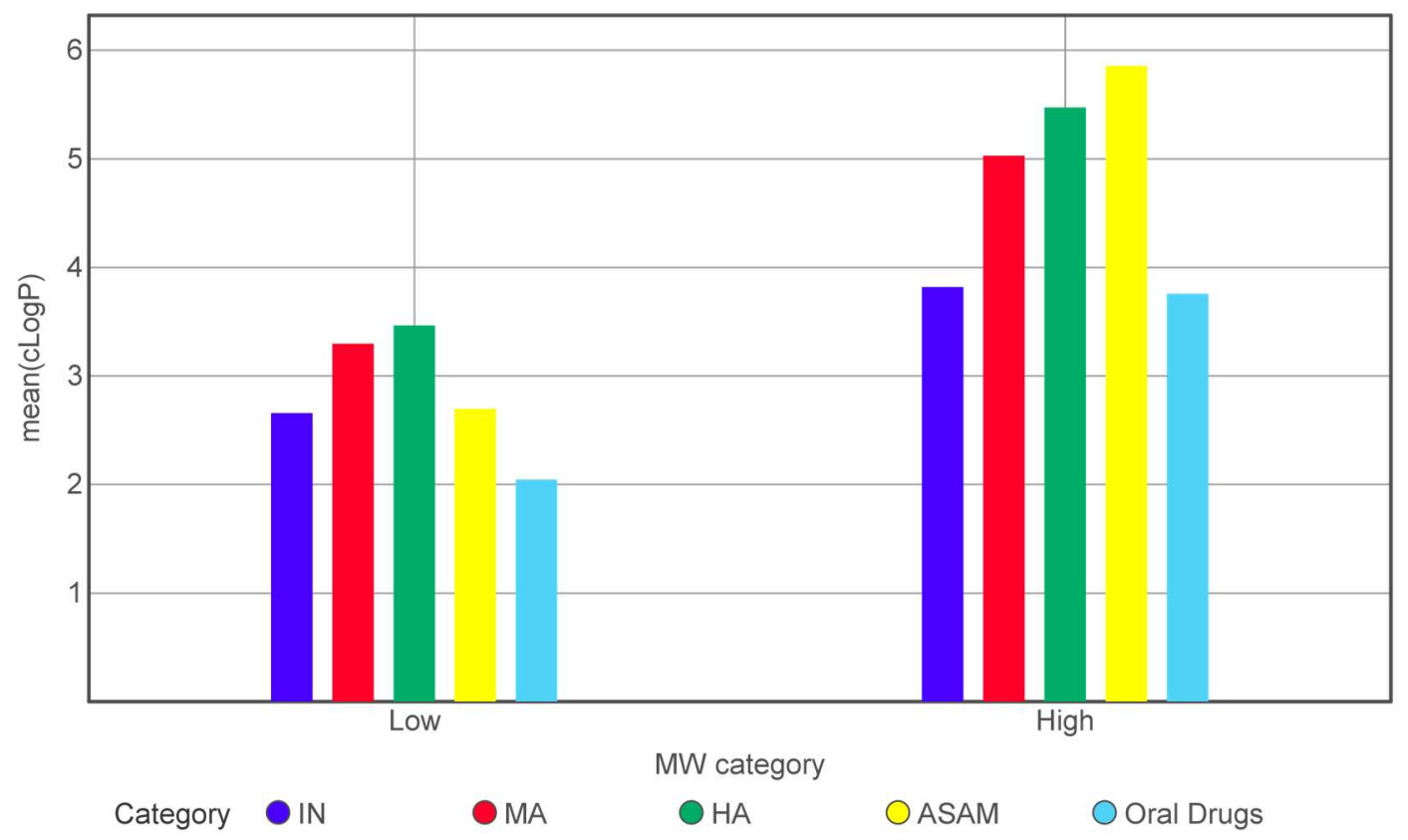

Figure S4: Distribution of mean of HBA among low (MW < 500) and high (MW > 500 Da) molecular weight compounds within the IN, MA, HA, ASAM, and oral drugs categories.

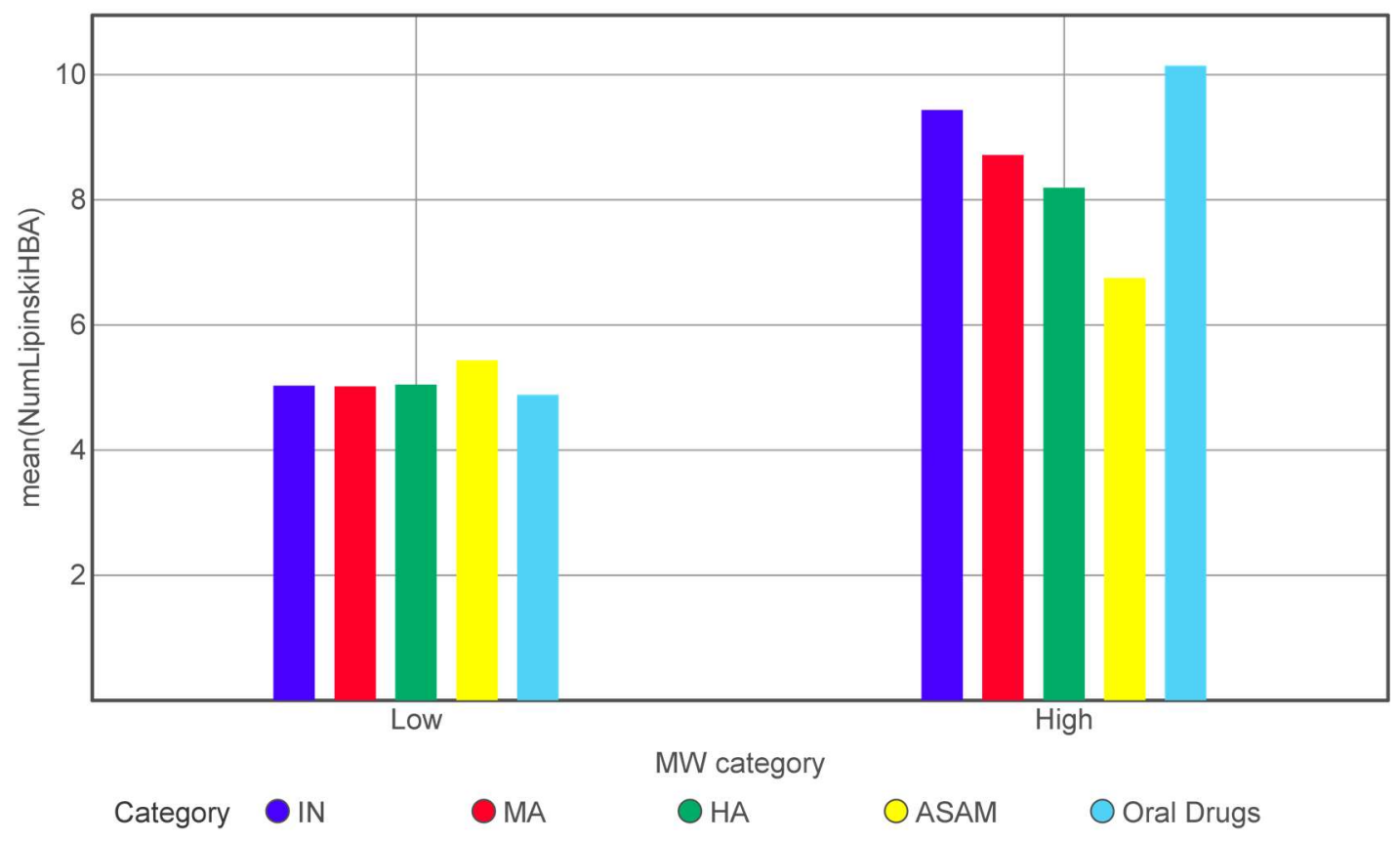


Figure S5: Distribution of mean of HBD among low (MW < 500) and high (MW > 500 Da) molecular weight compounds within the IN, MA, HA, ASAM, and oral drugs categories.

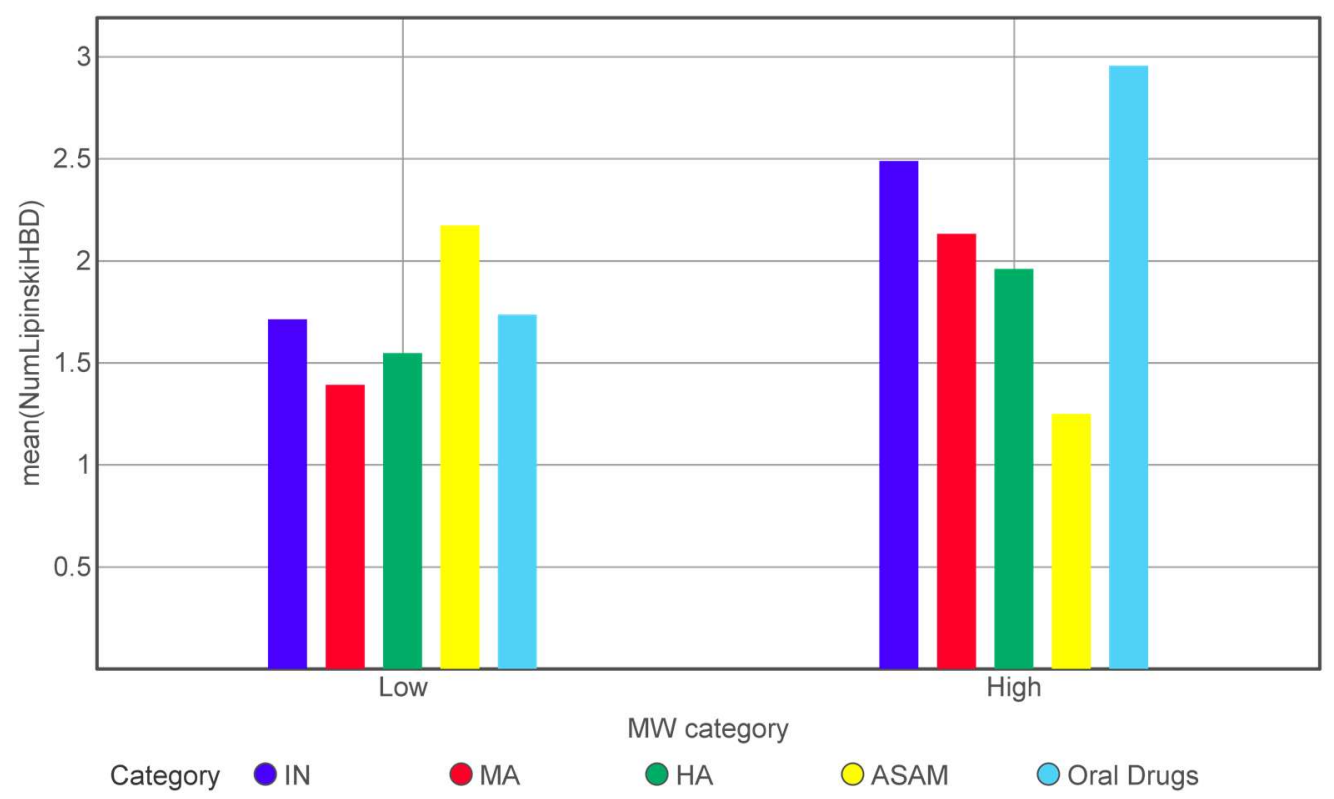

Figure S6: Distribution of mean of TPSA among low (MW < 500) and high (MW > $500 \mathrm{Da})$ molecular weight compounds within the IN, MA, HA, ASAM, and oral drugs categories.

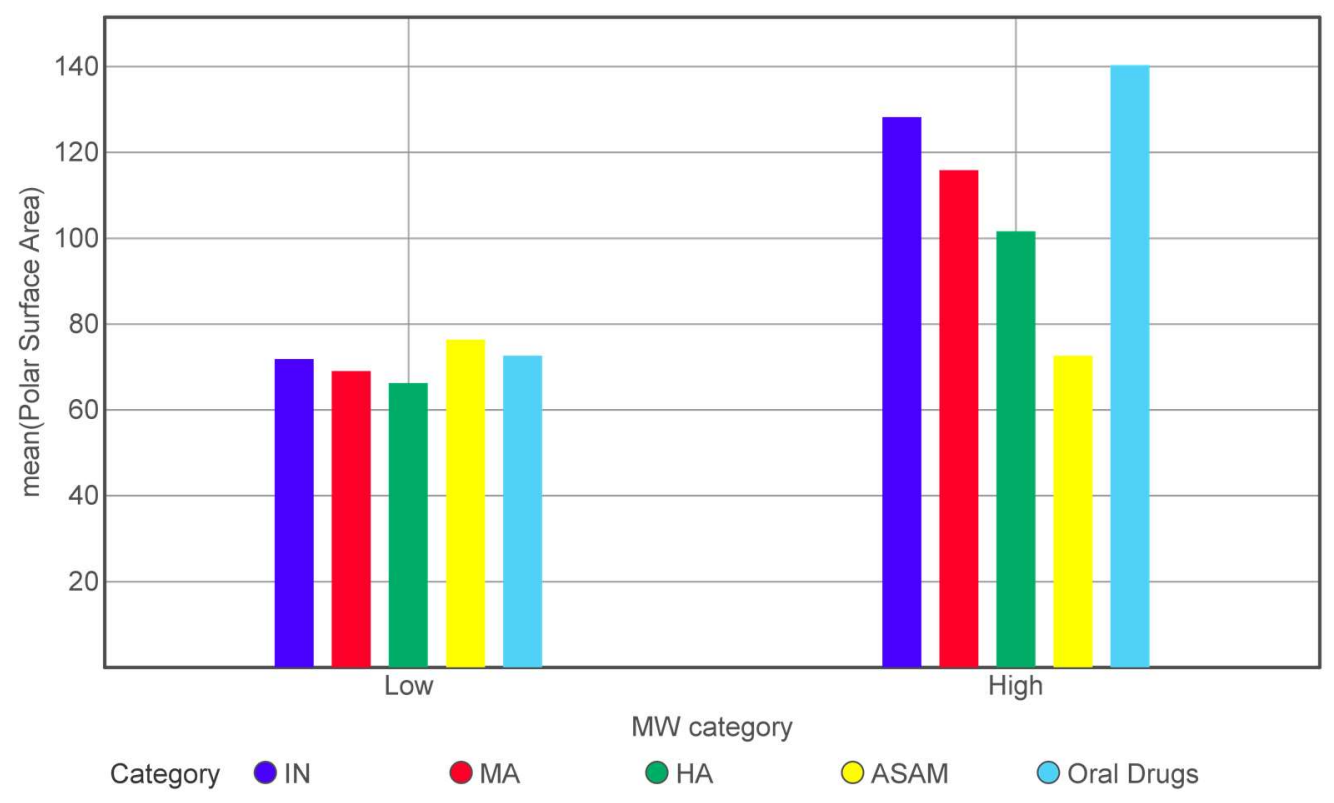


Figure S7: Distribution of mean of \#RB among low (MW < 500) and high (MW > $500 \mathrm{Da})$ molecular weight compounds within the IN, MA, HA, ASAM, and oral drugs categories

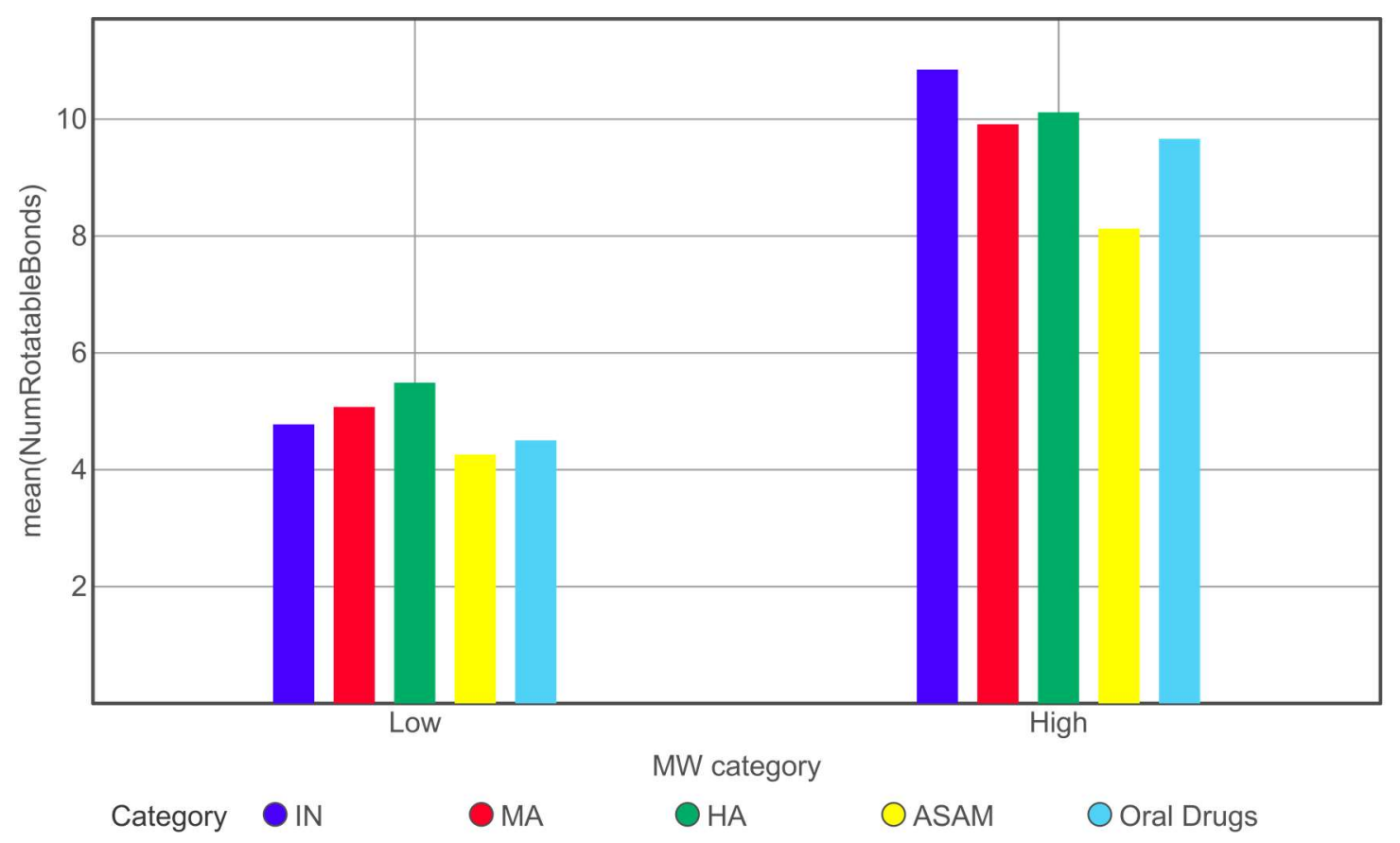

Figure S8: Distribution of mean of \#Ar among low (MW < 500) and high (MW > 500 Da) molecular weight compounds within the IN, MA, HA, ASAM, and oral drugs categories.

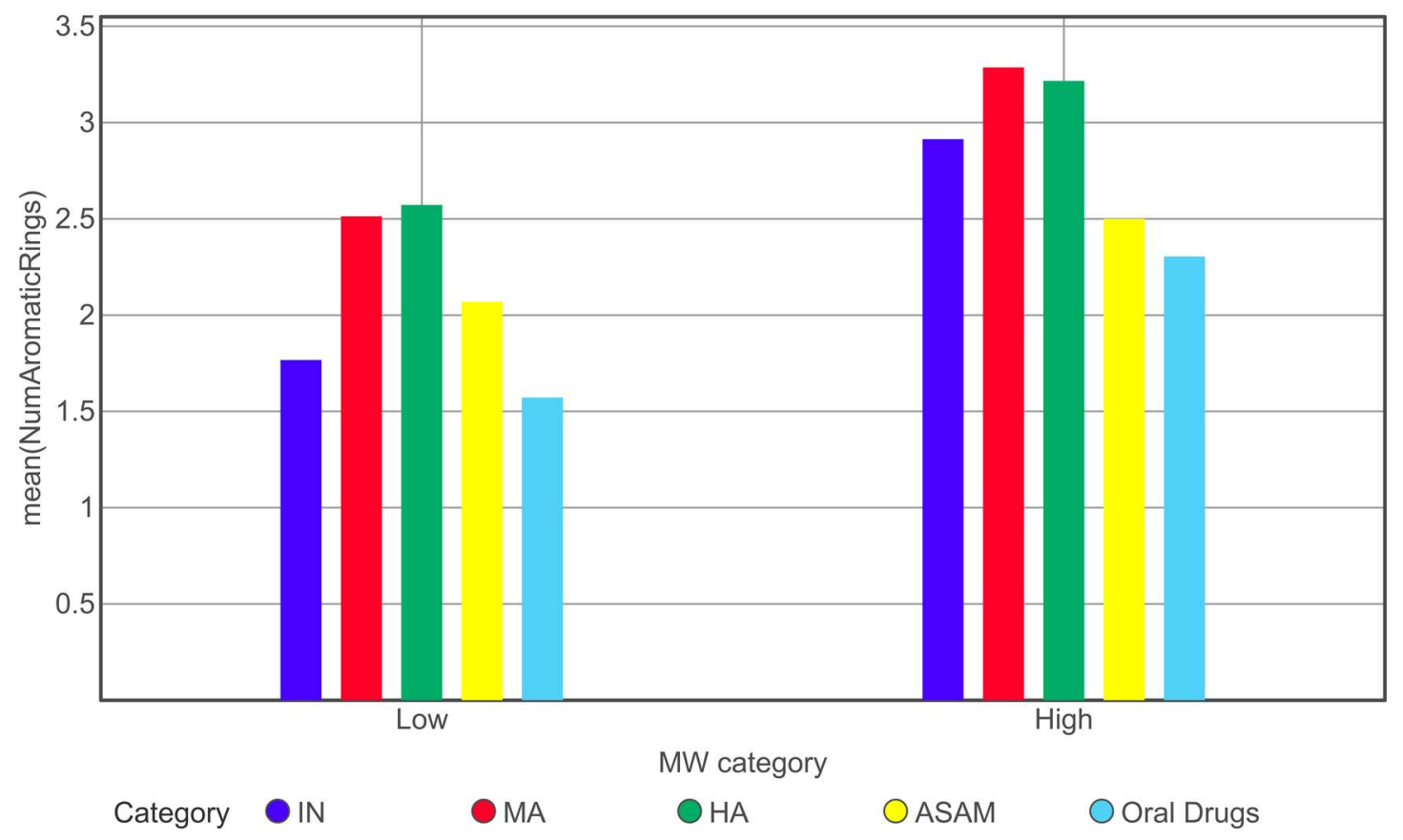


Figure S9: Distribution of mean of \#CarboAr among low $(\mathrm{MW}<500)$ and high $(\mathrm{MW}>500 \mathrm{Da})$ molecular weight compounds within the IN, MA, HA, ASAM, and oral drugs categories.

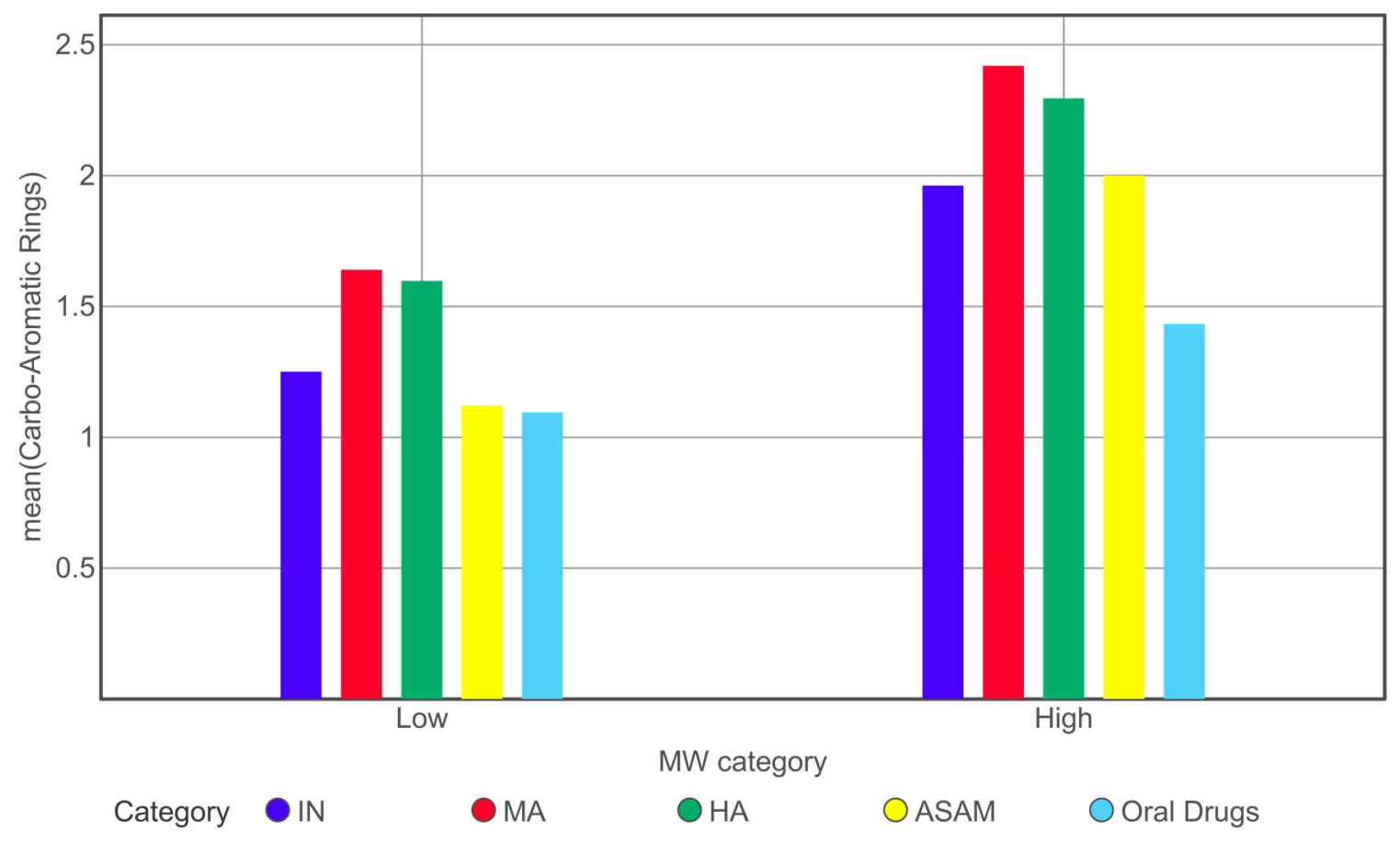

Figure S10: Distribution of mean of \#HetAr among low $(\mathrm{MW}<500)$ and high $(\mathrm{MW}>500 \mathrm{Da})$ molecular weight compounds within the IN, MA, HA, ASAM, and oral drugs categories.

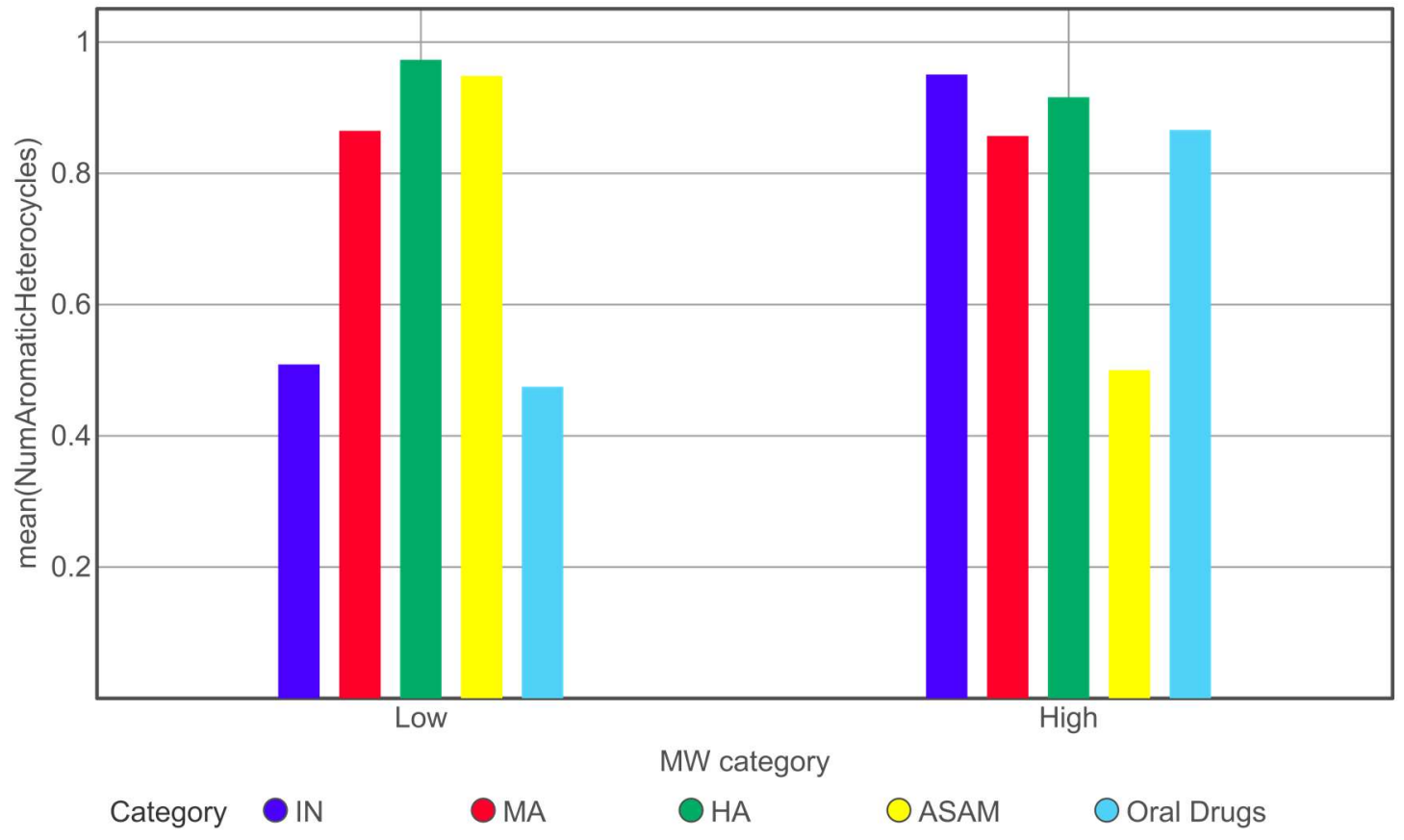


Figure S11: Distribution of mean of \#ArN among low $(\mathrm{MW}<500)$ and high $(\mathrm{MW}>500 \mathrm{Da})$ molecular weight compounds within the IN, MA, HA, ASAM, and oral drugs categories.

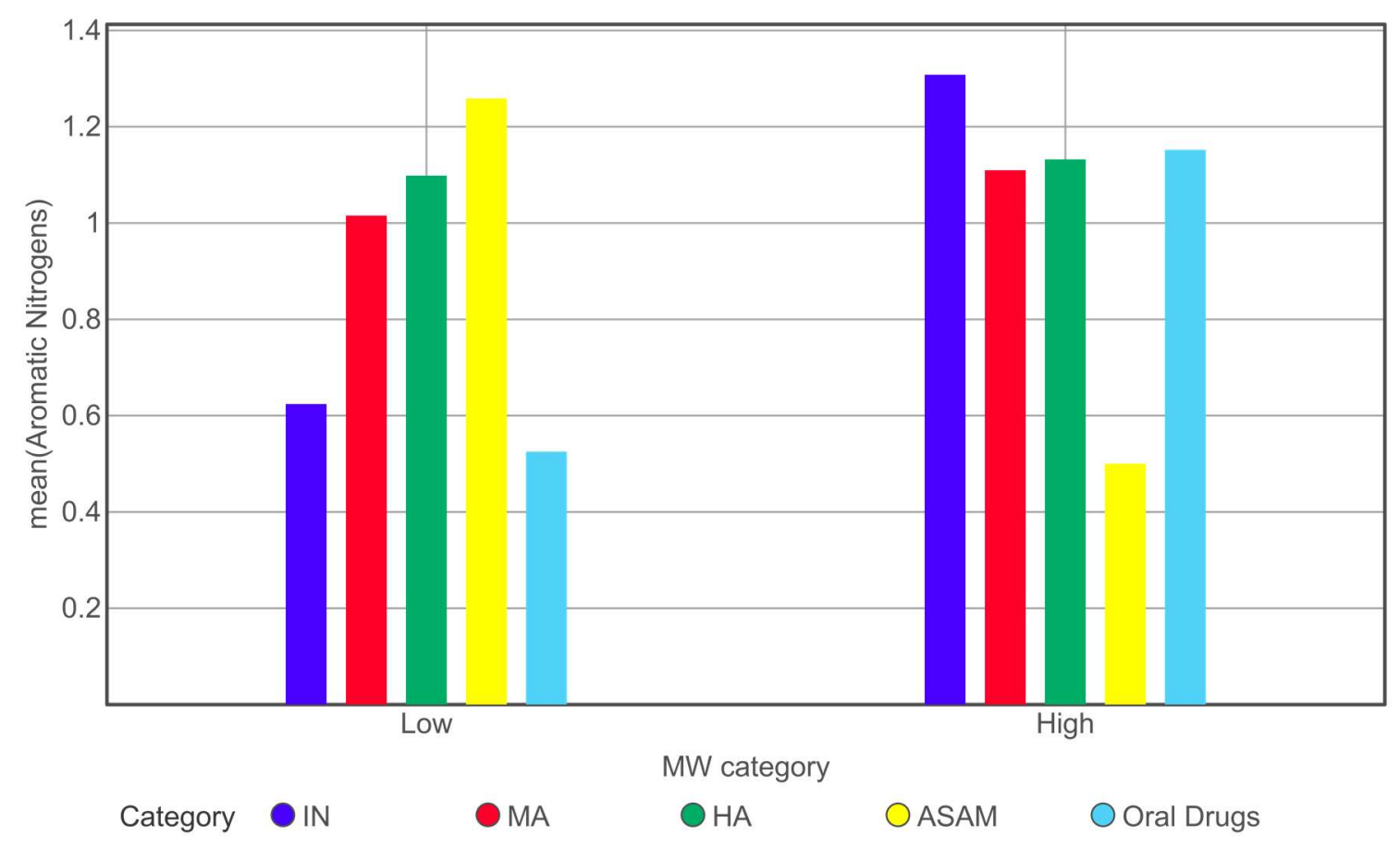

Figure S12: Distribution of mean of Fsp3 among low (MW < 500) and high (MW $>500 \mathrm{Da})$ molecular weight compounds within the IN, MA, HA, ASAM, and oral drugs categories.

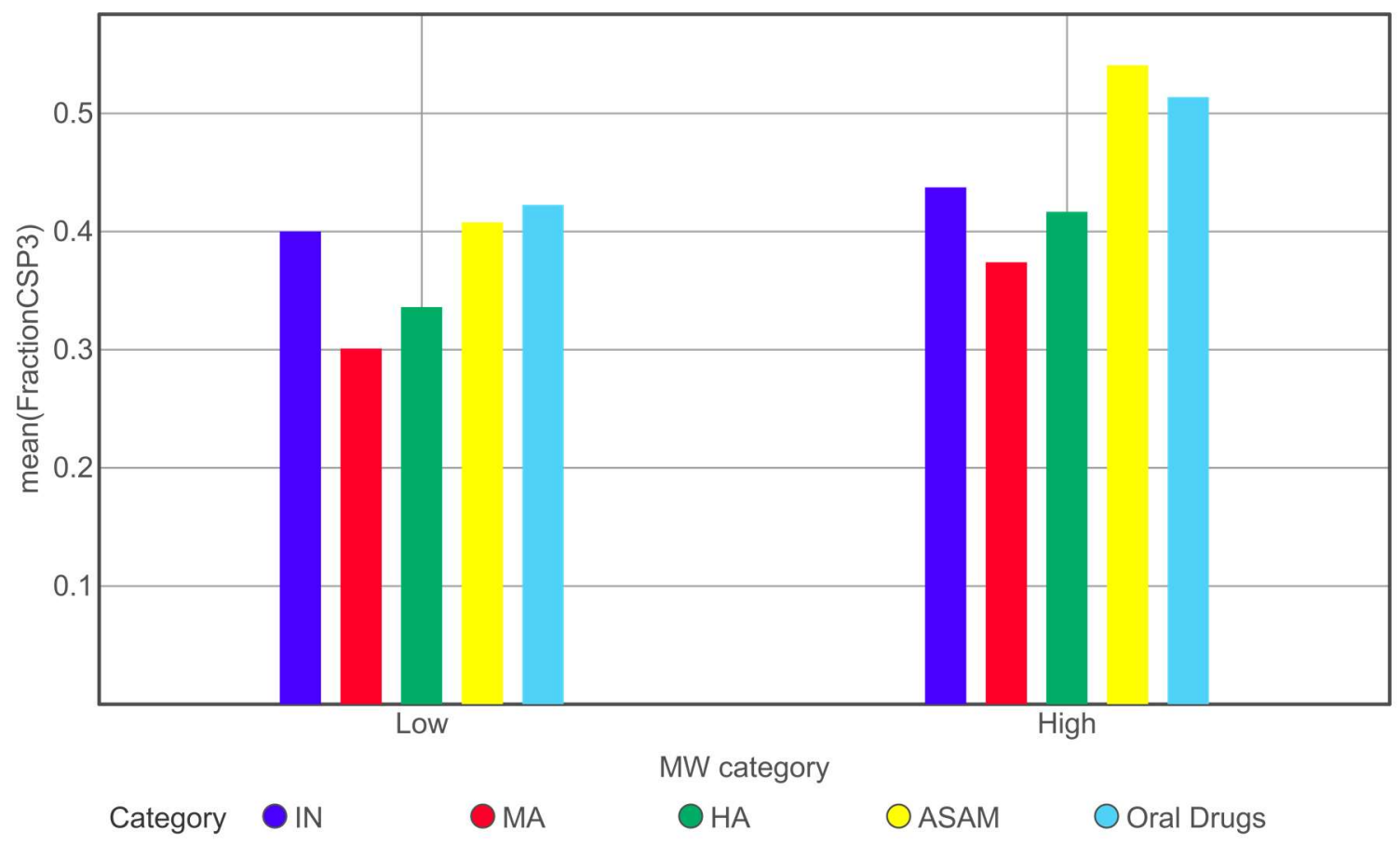


Figure S13: Distribution of mean of \#BaN among low $(\mathrm{MW}<500)$ and high $(\mathrm{MW}>500 \mathrm{Da})$ molecular weight compounds within the IN, MA, HA, ASAM, and oral drugs categories.

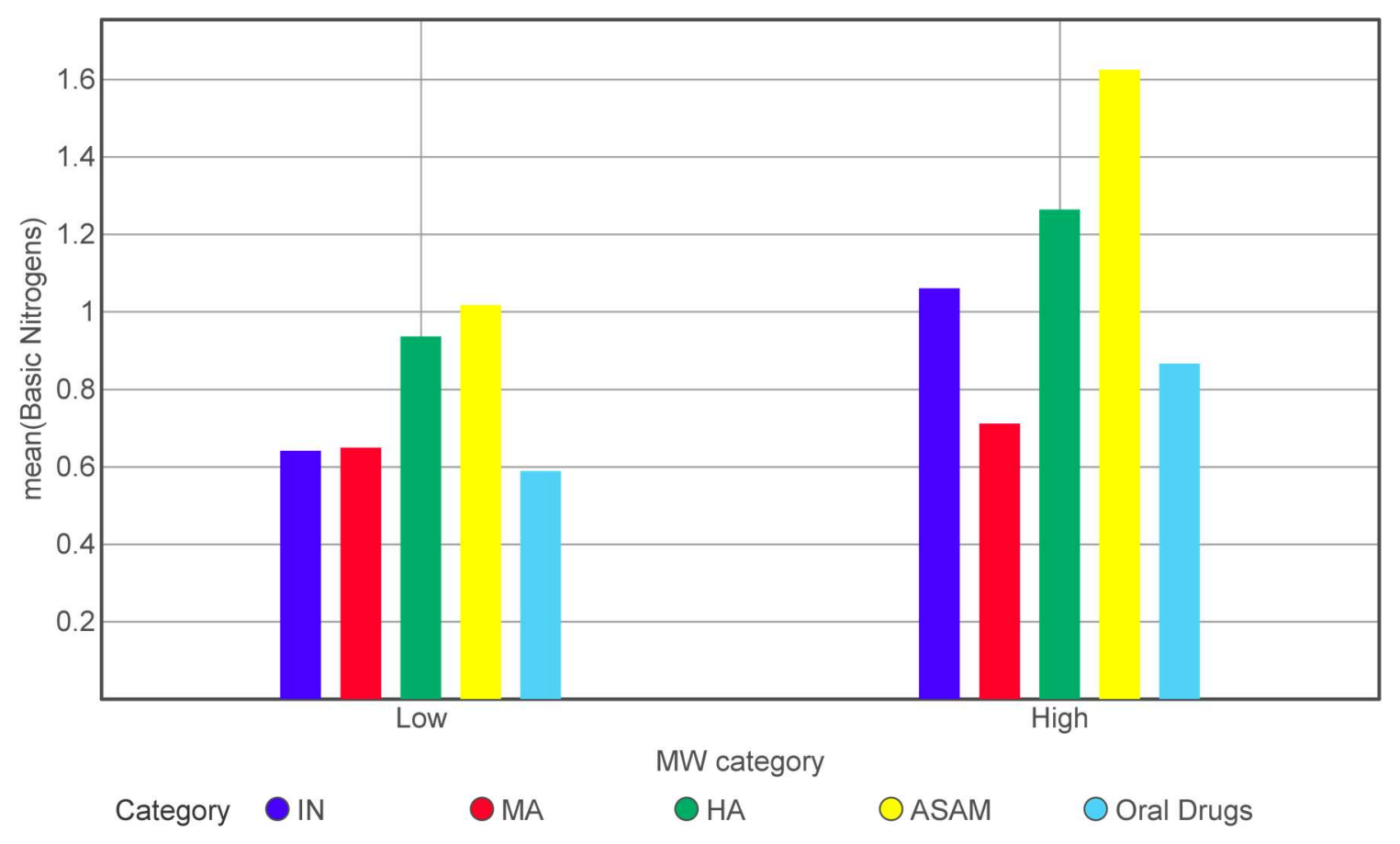


Figure S14: Plot of s-RB vs s-TPSA descriptors displaying ASAM molecules with high polarity not complying to Guideline-2.

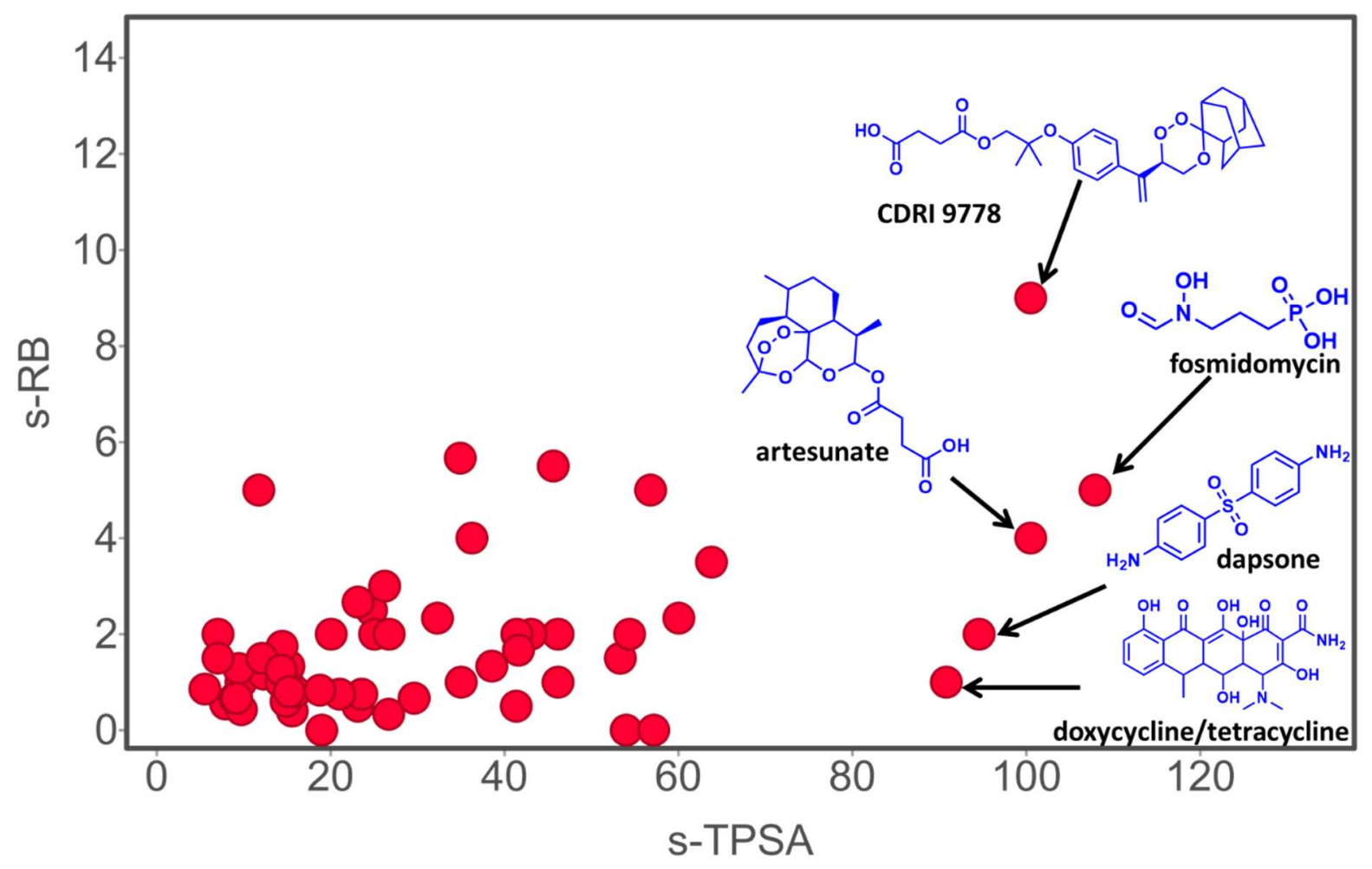




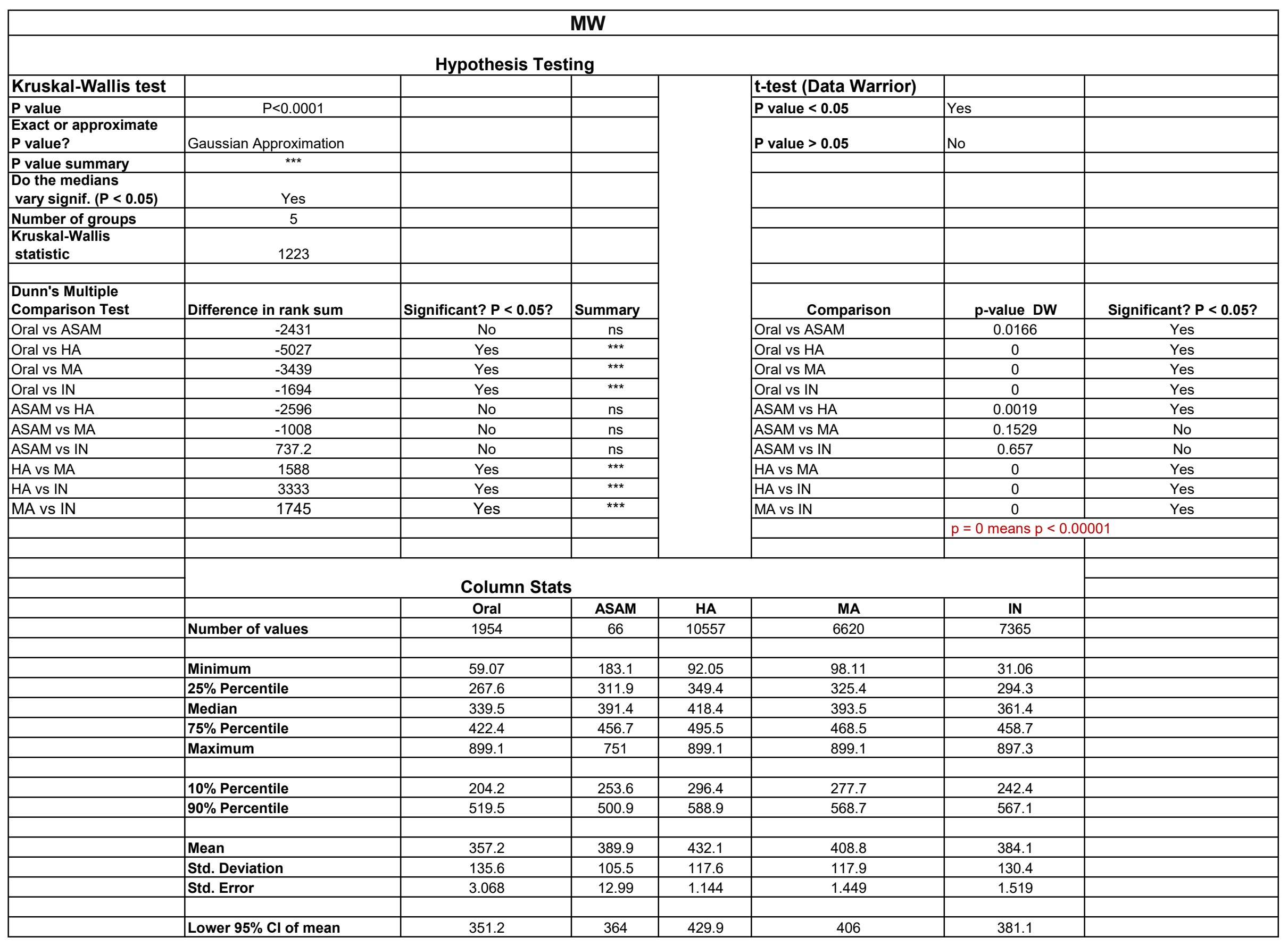




\begin{tabular}{|l|l|c|c|c|c|c|c|}
\hline & Upper 95\% Cl of mean & 363.2 & 415.8 & 434.4 & 411.6 & 387 \\
\hline & & & & & & \\
\hline & Skewness & 1.079 & 0.9787 & 0.8112 & 0.8843 & 0.7239 \\
\hline & Kurtosis & 2.054 & 2.463 & 0.8932 & 1.139 & 0.5411 \\
\hline & & & & & & \\
\hline \\
\hline
\end{tabular}


clogP

\begin{tabular}{|c|c|c|c|c|c|c|c|}
\hline \multicolumn{8}{|c|}{ Hypothesis Testing } \\
\hline Kruskal-Wallis test & & & & & & t-test (Data Warrior) & \\
\hline P value & $P<0.0001$ & & & & & $\mathrm{P}$ value $<0.05$ & Yes \\
\hline $\begin{array}{l}\text { Exact or approximate } \\
\text { P value? }\end{array}$ & Gaussian Approximation & & & & & $P$ value $>0.05$ & No \\
\hline$P$ value summary & *** & & & & & & \\
\hline $\begin{array}{l}\text { Do the medians vary } \\
\text { signif. }(\mathrm{P}<0.05)\end{array}$ & Yes & & & & & & \\
\hline Number of groups & 5 & & & & & & \\
\hline Kruskal-Wallis statistic & 1673 & & & & & & \\
\hline \begin{tabular}{|l|} 
Dunn's Multiple \\
Comparison Test \\
\end{tabular} & $\begin{array}{l}\text { Difference in } \\
\text { rank sum }\end{array}$ & Significant? $\mathrm{P}<0.05$ ? & Summary & & Comparison & p-value & Significant? $\mathrm{P}<0.05 ?$ \\
\hline Oral vs ASAM & -2632 & No & ns & & Oral vs ASAM & 0.0044 & Yes \\
\hline Oral vs HA & -5979 & Yes & 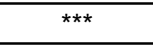 & & Oral vs HA & 0 & Yes \\
\hline Oral vs MA & -4592 & Yes & $* * *$ & & Oral vs MA & 0 & Yes \\
\hline Oral vs IN & -2203 & Yes & *** & & Oral vs IN & 0 & Yes \\
\hline ASAM vs HA & -3347 & Yes & ** & & ASAM vs HA & 0.0029 & Yes \\
\hline ASAM vs MA & -1959 & No & ns & & ASAM vs MA & 0.0634 & No \\
\hline ASAM vs IN & 429.5 & No & ns & & ASAM vs IN & 0.4543 & No \\
\hline HA vs MA & 1388 & Yes & *** & & HA vs MA & 0 & Yes \\
\hline HA vs IN & 3777 & Yes & *** & & HA vs IN & 0 & Yes \\
\hline MA vs IN & 2389 & Yes & $* * *$ & & MA vs IN & 0 & Yes \\
\hline & & & & & & $p=0$ means $p<0.00001$ & \\
\hline & & & & & & & \\
\hline & & & & & & & \\
\hline & & & & & & & \\
\hline & & Colun & & & & & \\
\hline & & Oral & ASAM & HA & MA & IN & \\
\hline & \begin{tabular}{|l|} 
Number of values \\
\end{tabular} & 1954 & 66 & 10557 & 6620 & 7365 & \\
\hline & & & & & & & \\
\hline & \begin{tabular}{|l|} 
Minimum \\
\end{tabular} & -9.713 & -4.142 & -12.89 & -10.43 & -11.85 & \\
\hline & 25\% Percentile & 0.9627 & 1.958 & 2.802 & 2.553 & 1.702 & \\
\hline & \begin{tabular}{|l|} 
Median \\
\end{tabular} & 2.439 & 3.133 & 3.972 & 3.603 & 3.004 & \\
\hline & 75\% Percentile & 3.741 & 4.25 & 5.172 & 4.688 & 4.313 & \\
\hline & Maximum & 22.09 & 8.732 & 14.72 & 12.35 & 18.04 & \\
\hline & & & & & & & \\
\hline & $10 \%$ Percentile & -0.8722 & 0.3404 & 1.485 & 1.472 & 0.1952 & \\
\hline & $90 \%$ Percentile & 4.858 & 5.542 & 6.431 & 5.779 & 5.645 & \\
\hline & \begin{tabular}{|l|} 
Mean \\
\end{tabular} & 2.24 & 3.078 & 3.943 & 3.606 & 2.867 & \\
\hline
\end{tabular}




\begin{tabular}{|l|c|c|c|c|c|}
\hline Std. Deviation & 2.516 & 2.264 & 2.114 & 2.045 & 2.505 \\
\hline Std. Error & 0.05692 & 0.2787 & 0.02057 & 0.02513 & 0.02919 \\
\hline & & & & & \\
\hline Lower 95\% Cl of mean & 2.128 & 2.521 & 3.903 & 3.557 & 2.809 \\
\hline Upper 95\% CI of mean & 2.351 & 3.634 & 3.983 & 3.655 & 2.924 \\
\hline & & & & & -0.8457 \\
\hline Skewness & -0.06683 & -0.3104 & -0.3555 & -0.4811 & 3.896 \\
\hline Kurtosis & 4.364 & 1.49 & 2.08 & 5.077 & 21113 \\
\hline & & & & & 67 \\
\hline Sum & 4377 & 203.1 & 41626 & 23871 & 4 \\
\hline Outliers(Data Warrior) & 313 & 304 & 403 & 4 & \\
\hline
\end{tabular}




\begin{tabular}{|c|c|c|c|c|c|c|c|}
\hline & & & & & HBA & & \\
\hline & Hypothesis & Testing & & & & & \\
\hline Kruskal-Wallis tes & & & & & t-test (Data Warrior) & & \\
\hline$P$ value & $P<0.0001$ & & & & $P$ value $<0.05$ & Yes & \\
\hline $\begin{array}{l}\text { Exact or } \\
\text { approximate } \\
\text { P value? }\end{array}$ & Gaussian Approximation & & & & $P$ value $>0.05$ & No & \\
\hline$P$ value summary & $* * *$ & & & & & & \\
\hline $\begin{array}{l}\text { Do the medians } \\
\text { vary signif. ( }<< \\
0.05)\end{array}$ & Yes & & & & & & \\
\hline Number of groups & 5 & & & & & & \\
\hline $\begin{array}{l}\text { Kruskal-Wallis } \\
\text { statistic }\end{array}$ & 81.64 & & & & & & \\
\hline $\begin{array}{l}\text { Dunn's Multiple } \\
\text { Comparison Test }\end{array}$ & $\begin{array}{l}\text { Difference in } \\
\text { rank sum }\end{array}$ & \begin{tabular}{|l|} 
Significant? \\
$\mathrm{P}<0.05 ?$
\end{tabular} & Summary & & Comparison & p-value DW & $\begin{array}{c}\text { Significant? } \\
P<0.05 ?\end{array}$ \\
\hline Oral vs ASAM & -1193 & No & $\mathrm{ns}$ & & Oral vs ASAM & 0.6957 & No \\
\hline Oral vs HA & -1608 & Yes & 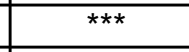 & & Oral vs HA & 0 & Yes \\
\hline Oral vs MA & -1032 & Yes & *** & & Oral vs MA & 0.012 & Yes \\
\hline Oral vs IN & -1227 & Yes & 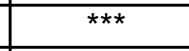 & & Oral vs IN & 0 & Yes \\
\hline ASAM vs HA & -414.5 & No & ns & & ASAM vs HA & 0.4399 & No \\
\hline ASAM vs MA & 160.7 & No & $\mathrm{ns}$ & & ASAM vs MA & 0.7415 & No \\
\hline ASAM vs IN & -34.16 & No & $\mathrm{ns}$ & & ASAM vs IN & 0.3894 & No \\
\hline HA vs MA & 575.1 & $\overline{\text { Yes }}$ & *** & & HA vs MA & 0.0034 & $\overline{\text { Yes }}$ \\
\hline HA vs IN & 380.3 & Yes & ** & & HA vs IN & 0.5518 & No \\
\hline MA vs IN & -194.8 & No & $\mathrm{ns}$ & & MA vs IN & 0.0021 & Yes \\
\hline & & & & & & $p=0$ means $p$ & 001 \\
\hline & & & & & & & \\
\hline & & & Column Sta & & & & \\
\hline & & & & & & & \\
\hline & & Oral & ASAM & HA & MA & IN & \\
\hline & Number of values & 1954 & 66 & 10557 & 6620 & 7365 & \\
\hline & & & & & & & \\
\hline & Minimum & 0 & 2 & 0 & 0 & 0 & \\
\hline & 25\% Percentile & 3 & 4 & 4 & 4 & 4 & \\
\hline & Median & 5 & 5.5 & 6 & 5 & 5 & \\
\hline & $75 \%$ Percentile & 7 & 6.25 & 7 & 7 & 7 & \\
\hline
\end{tabular}




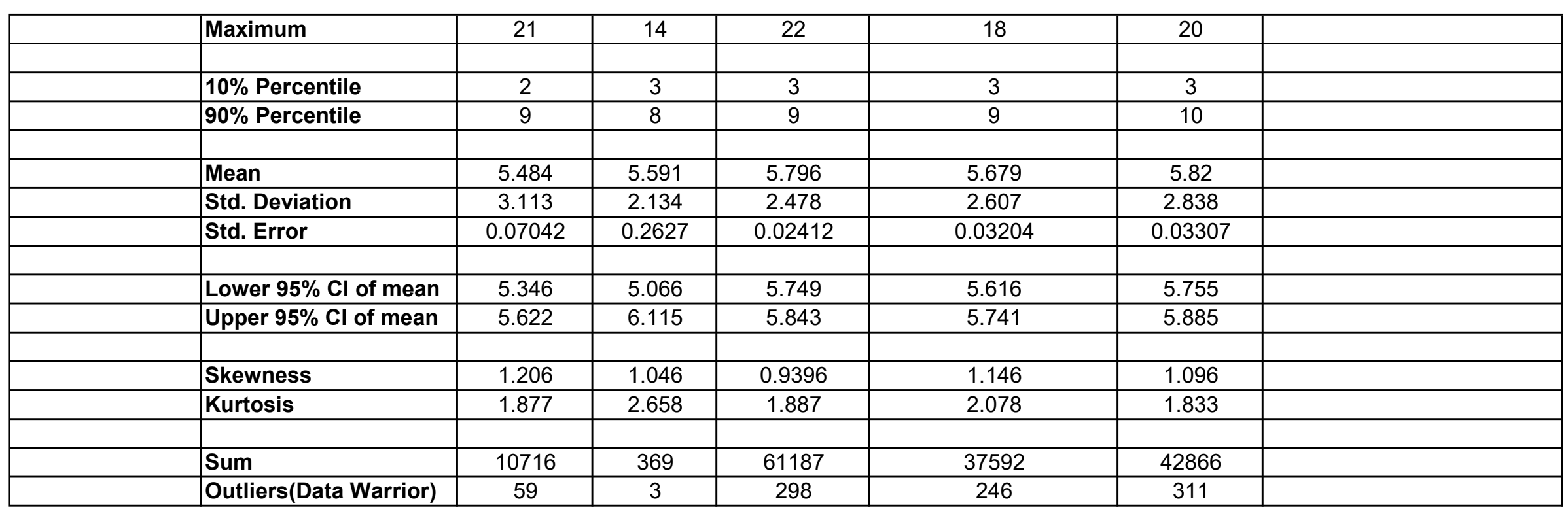




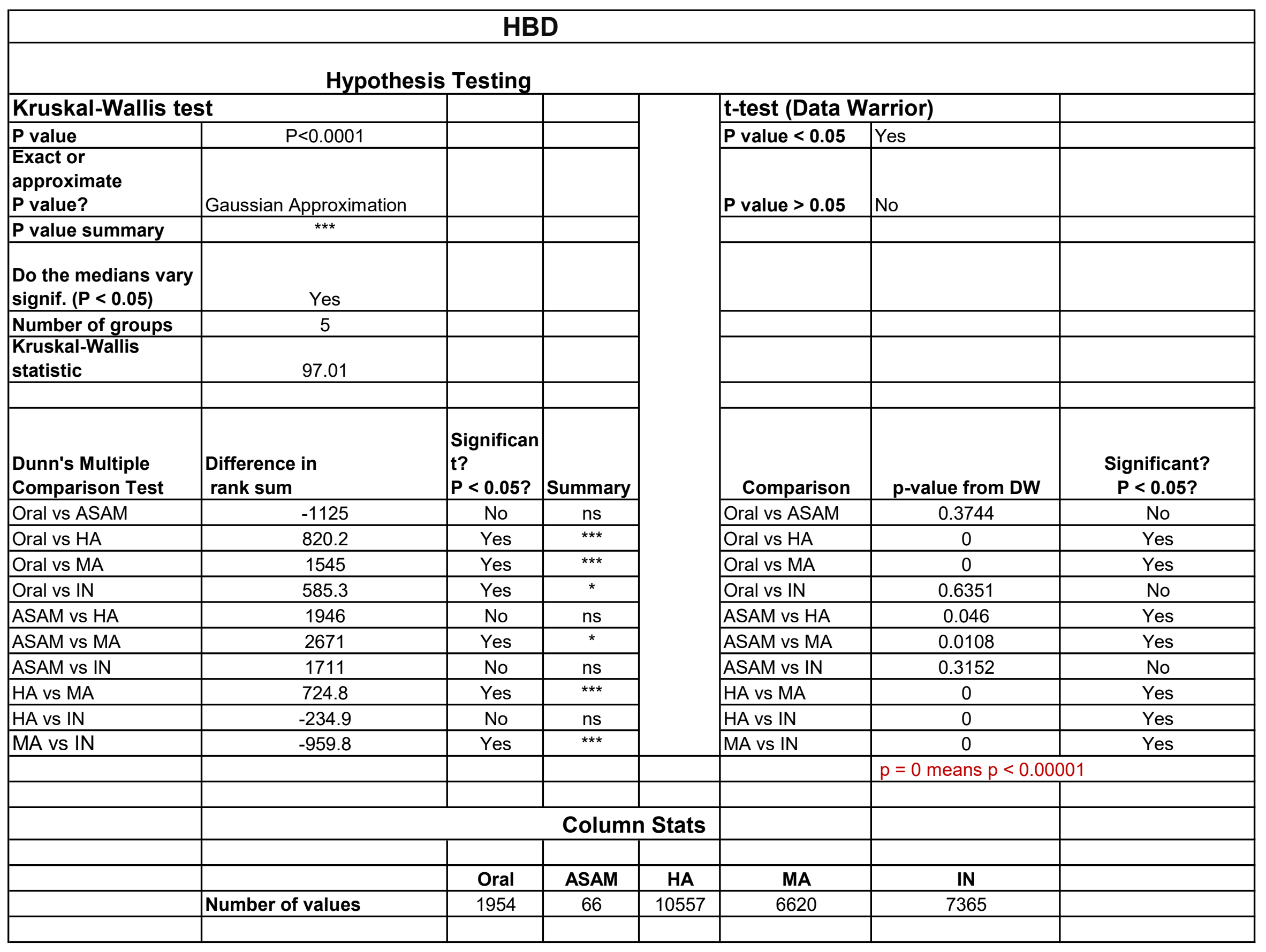




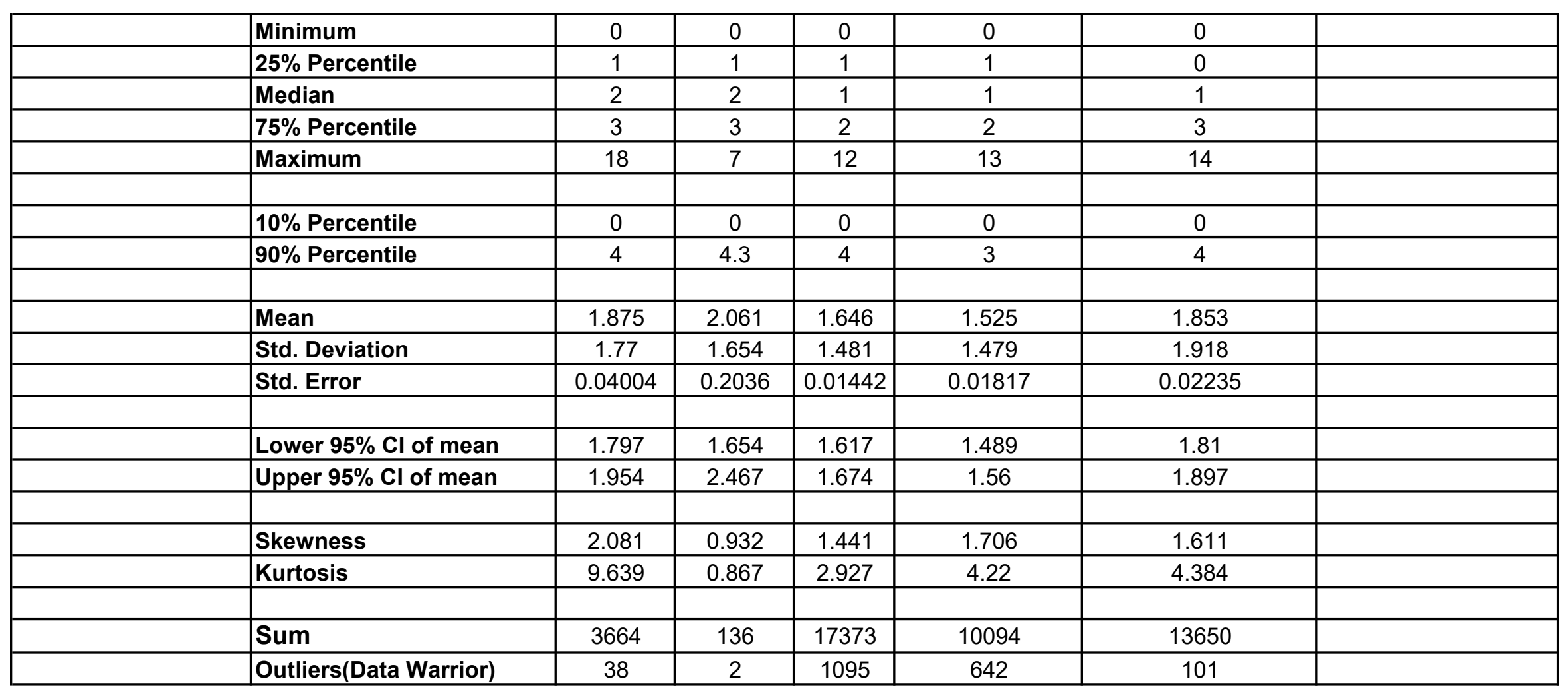


TPSA

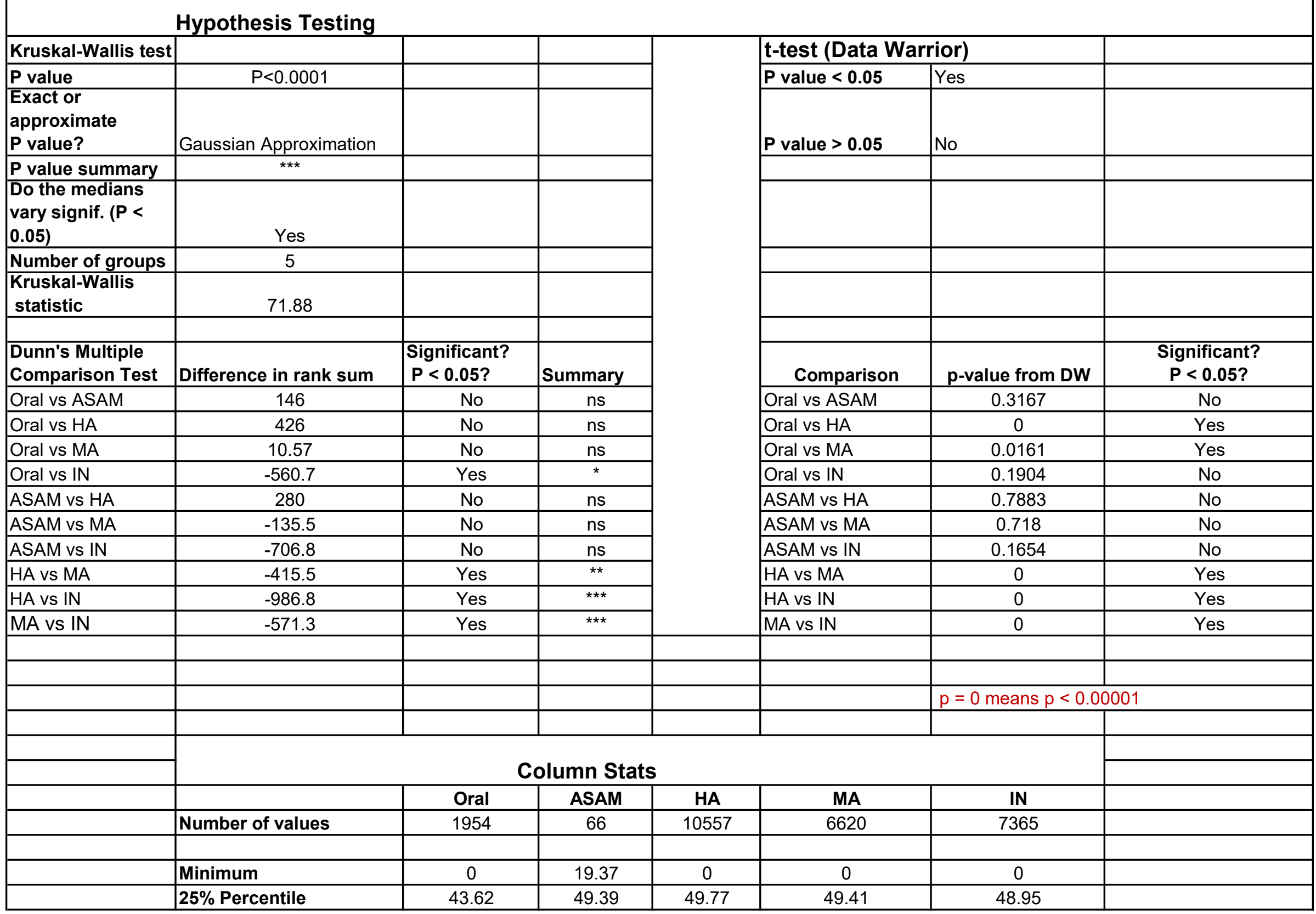




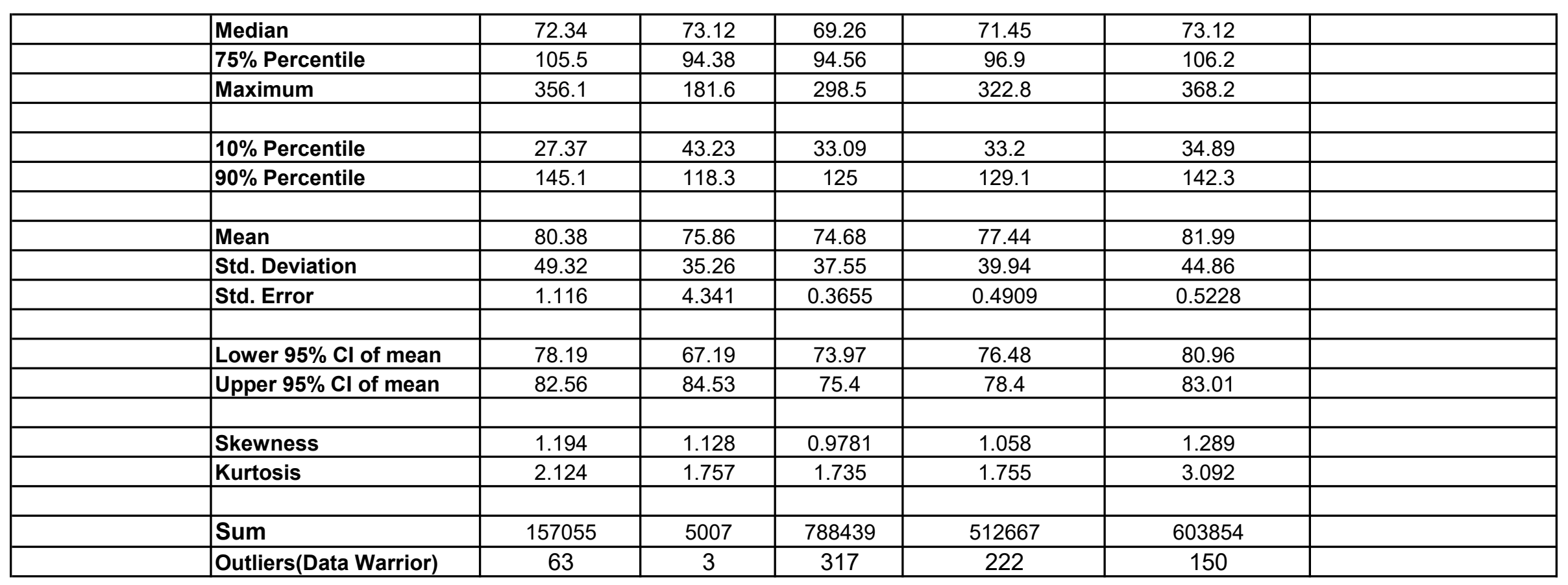




\begin{tabular}{|c|c|c|c|c|c|c|c|}
\hline \multicolumn{8}{|c|}{ \#RB } \\
\hline \multicolumn{8}{|c|}{ Hypothesis Testing } \\
\hline Kruskal-Wallis test & & & & & \multicolumn{2}{|c|}{ t-test (Data Warrior) } & \\
\hline$P$ value & $P<0.0001$ & & & & $P$ value $<0.05$ & Yes & \\
\hline $\begin{array}{l}\text { Exact or approximate } \\
\text { P value? }\end{array}$ & Gaussian Approximation & & & & $P$ value $>0.05$ & No & \\
\hline$P$ value summary & $* * *$ & & & & & & \\
\hline $\begin{array}{l}\text { Do the medians } \\
\text { vary signif. }(P<0.05)\end{array}$ & Yes & & & & & & \\
\hline Number of groups & 5 & & & & & & \\
\hline $\begin{array}{l}\text { Kruskal-Wallis } \\
\text { statistic }\end{array}$ & 423.8 & & & & & & \\
\hline $\begin{array}{l}\text { Dunn's Multiple } \\
\text { Comparison Test }\end{array}$ & Difference in rank sum & $\begin{array}{l}\text { Significant? } \\
\mathrm{P}<0.05 ?\end{array}$ & Summary & & Comparison & $p$-value from DW & Significant? $P<0.05$ ? \\
\hline Oral vs ASAM & 576.7 & No & ns & & Oral vs ASAM & 0.3543 & No \\
\hline Oral vs HA & -3059 & Yes & $* * *$ & & Oral vs HA & 0 & Yes \\
\hline Oral vs MA & -1770 & Yes & $* * *$ & & Oral vs MA & 0 & Yes \\
\hline Oral vs IN & -1238 & Yes & $* * *$ & & Oral vs IN & 0 & Yes \\
\hline ASAM vs HA & -3635 & Yes & ** & & ASAM vs HA & 0 & Yes \\
\hline ASAM vs MA & -2347 & No & ns & & ASAM vs MA & 0.0024 & Yes \\
\hline ASAM vs IN & -1815 & No & ns & & ASAM vs IN & 0.0042 & Yes \\
\hline HA vs MA & 1288 & Yes & $* * *$ & & HA vs MA & 0 & Yes \\
\hline HA vs IN & 1820 & Yes & $* * *$ & & HA vs IN & 0 & Yes \\
\hline MA vs IN & 532 & Yes & $\star * *$ & & MA vs IN & 0.3131 & No \\
\hline & & & & & & $p=0$ means $p<0.00$ & 001 \\
\hline & & & & & & & \\
\hline & & & & & & & \\
\hline & & & tats & & & & \\
\hline & & & & & & & \\
\hline & & Oral & ASAM & HA & MA & IN & \\
\hline & Number of values & 1954 & 66 & 10557 & 6620 & 7365 & \\
\hline & Minimum & 0 & 0 & 0 & 0 & 0 & \\
\hline & $25 \%$ Percentile & 3 & 2 & 4 & 3 & 3 & \\
\hline & Median & 4 & 4 & 6 & 5 & 5 & \\
\hline & $75 \%$ Percentile & 7 & 7 & 9 & 7 & 8 & \\
\hline & Maximum & 32 & 17 & 41 & 41 & 46 & \\
\hline
\end{tabular}




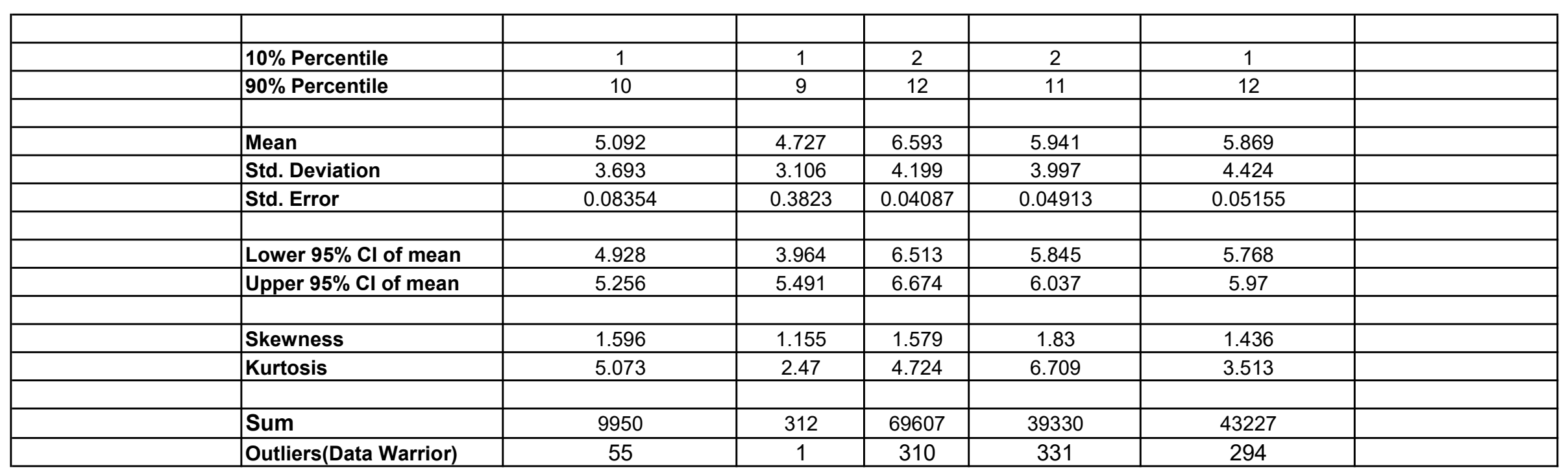




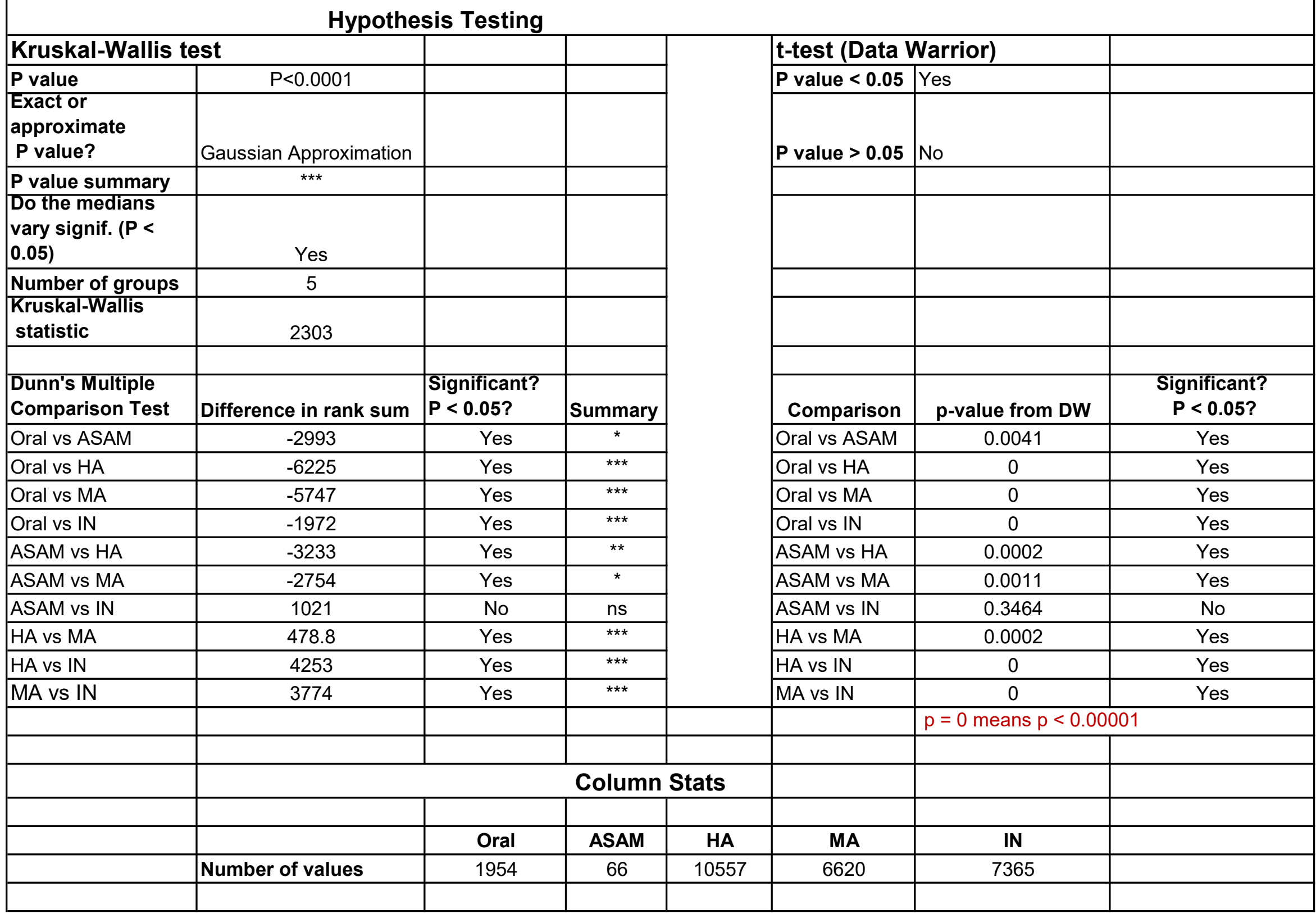




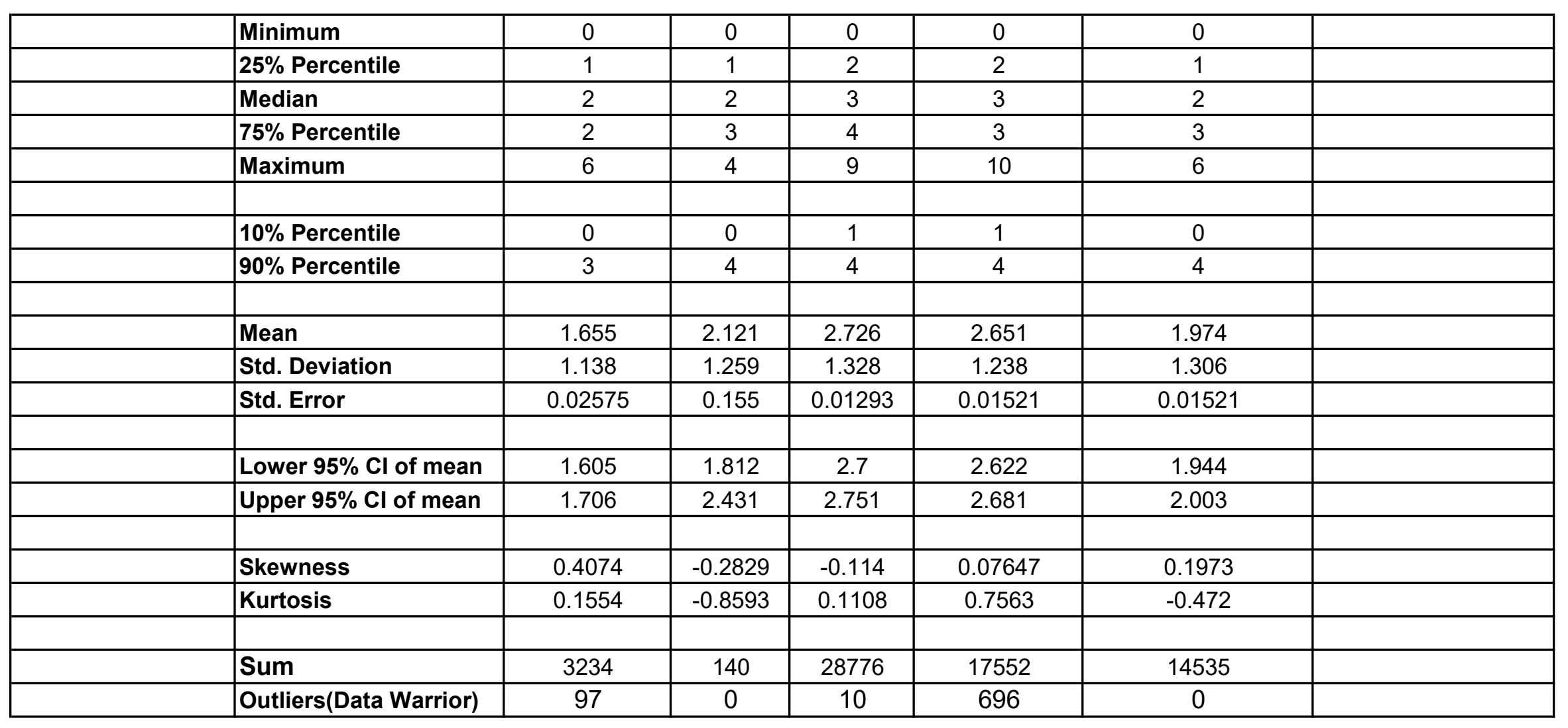




\section{Hypothesis Testing}

\begin{tabular}{|c|c|c|c|c|c|c|c|}
\hline Kruskal-Wallis test & & & & & t-test (Data Wa & rior) & \\
\hline$P$ value & $P<0.0001$ & & & & $P$ value $<0.05$ & Yes & \\
\hline \begin{tabular}{|l} 
Exact or \\
approximate \\
$P$ value?
\end{tabular} & Gaussian Approximation & & & & $P$ value $>0.05$ & No & \\
\hline $\begin{array}{l}\text { P value } \\
\text { summary }\end{array}$ & $* * *$ & & & & & & \\
\hline $\begin{array}{l}\text { Do the medians } \\
\text { vary signif. }(P< \\
0.05)\end{array}$ & Yes & & & & & & \\
\hline Number of groups & 5 & & & & & & \\
\hline $\begin{array}{l}\begin{array}{l}\text { Kruskal-Wallis } \\
\text { statistic }\end{array} \\
\end{array}$ & 1193 & & & & & & \\
\hline $\begin{array}{l}\text { Dunn's Multiple } \\
\text { Comparison Test }\end{array}$ & Difference in rank sum & $\begin{array}{l}\text { Significant? } \\
\mathrm{P}<0.05 ?\end{array}$ & Summary & & Comparison & $p$-value from DW & $\begin{array}{c}\text { Significant? } \\
P<0.05 ?\end{array}$ \\
\hline Oral vs ASAM & -504 & No & ns & & Oral vs ASAM & 0.3969 & No \\
\hline Oral vs HA & -4439 & Yes & 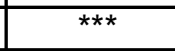 & & Oral vs HA & 0 & Yes \\
\hline$\overline{O r a l ~ v s ~ M A ~}$ & -4633 & Yes & $\overline{* \star \star \star}$ & & Oral vs MA & 0 & Yes \\
\hline Oral vs IN & -1794 & Yes & $\bar{*}^{* * *}$ & & Oral vs IN & 0 & Yes \\
\hline ASAM vs HA & -3935 & Yes & $* \star *$ & & ASAM vs HA & 0 & Yes \\
\hline ASAM vs MA & -4129 & Yes & *** & & ASAM vs MA & 0 & Yes \\
\hline ASAM vS IN & -1290 & No & $\mathrm{ns}$ & & $\overline{\text { ASAM vS IN }}$ & 0.165 & No \\
\hline HA vs MA & -194.6 & No & ns & & HA vs MA & 0.3315 & No \\
\hline HA vs IN & 2645 & Yes & 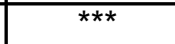 & & HA vs IN & 0 & Yes \\
\hline MA vs IN & 2840 & Yes & $\bar{*}^{* \star *}$ & & MA vs IN & 0 & Yes \\
\hline & & & & & & $p=0$ means $p<0.0$ & \\
\hline & & & & & & & \\
\hline & & & lumn Stats & & & & \\
\hline & & & & & & & \\
\hline & & Oral & ASAM & HA & MA & IN & \\
\hline & Number of values & 1954 & 66 & 10557 & 6620 & 7365 & \\
\hline & & & & & & & \\
\hline & Minimum & 0 & 0 & 0 & 0 & 0 & \\
\hline & $25 \%$ Percentile & 0 & 1 & 1 & 1 & 1 & \\
\hline
\end{tabular}




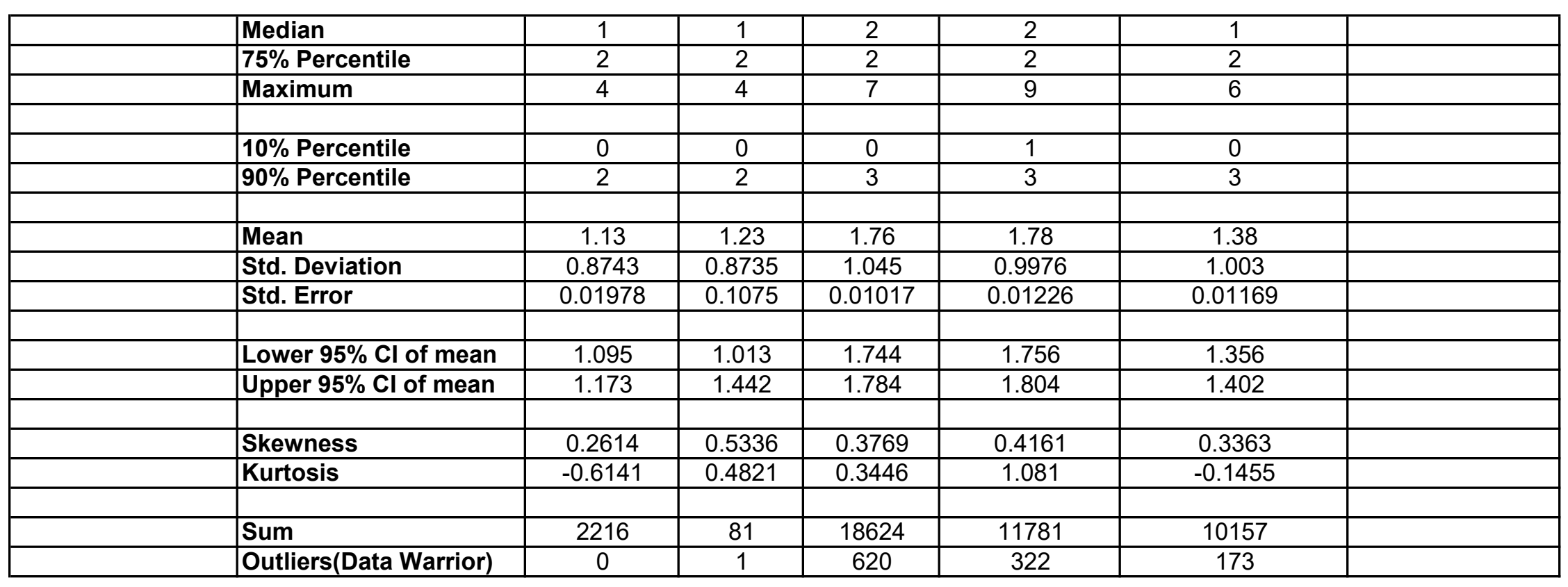




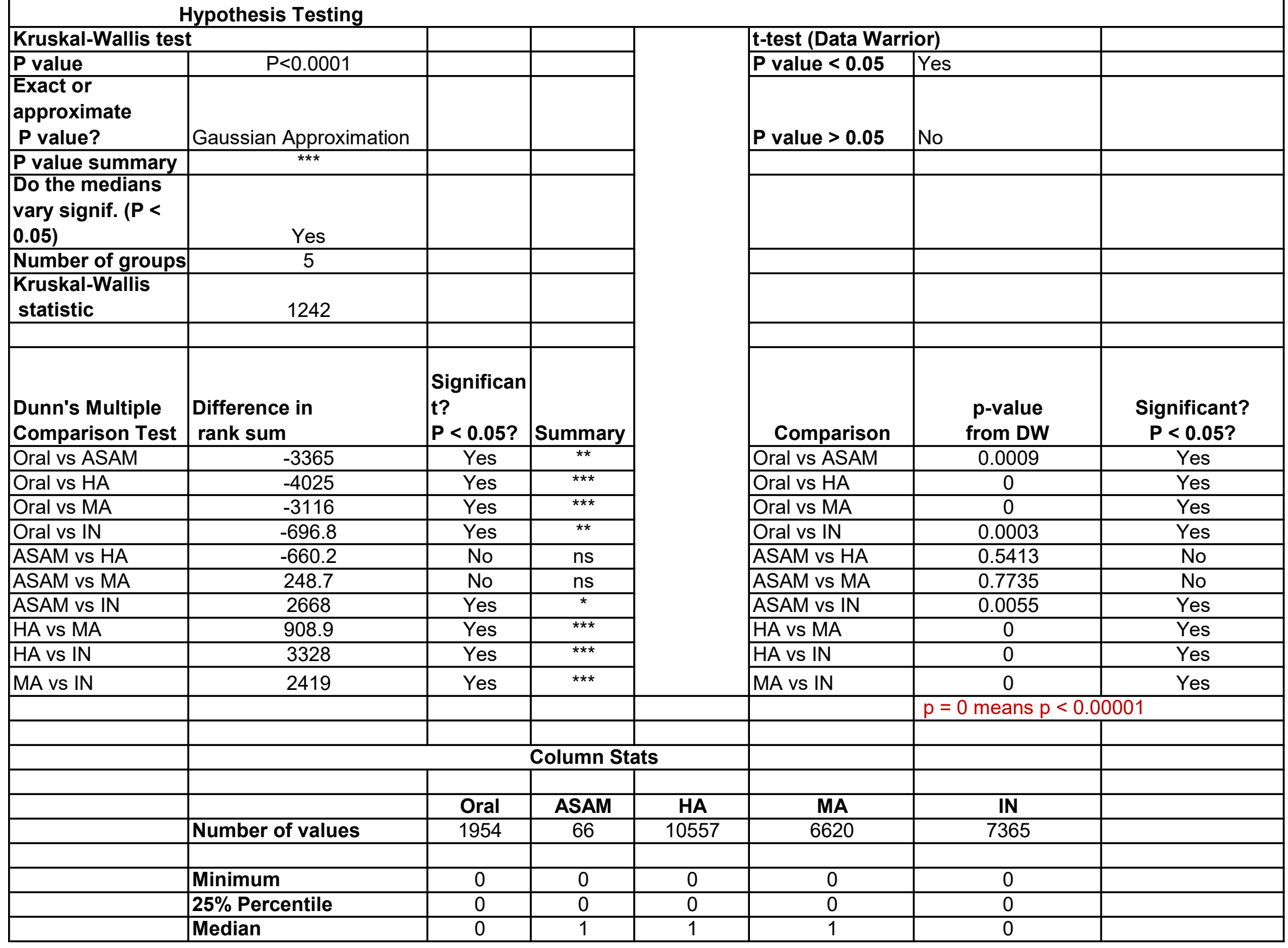




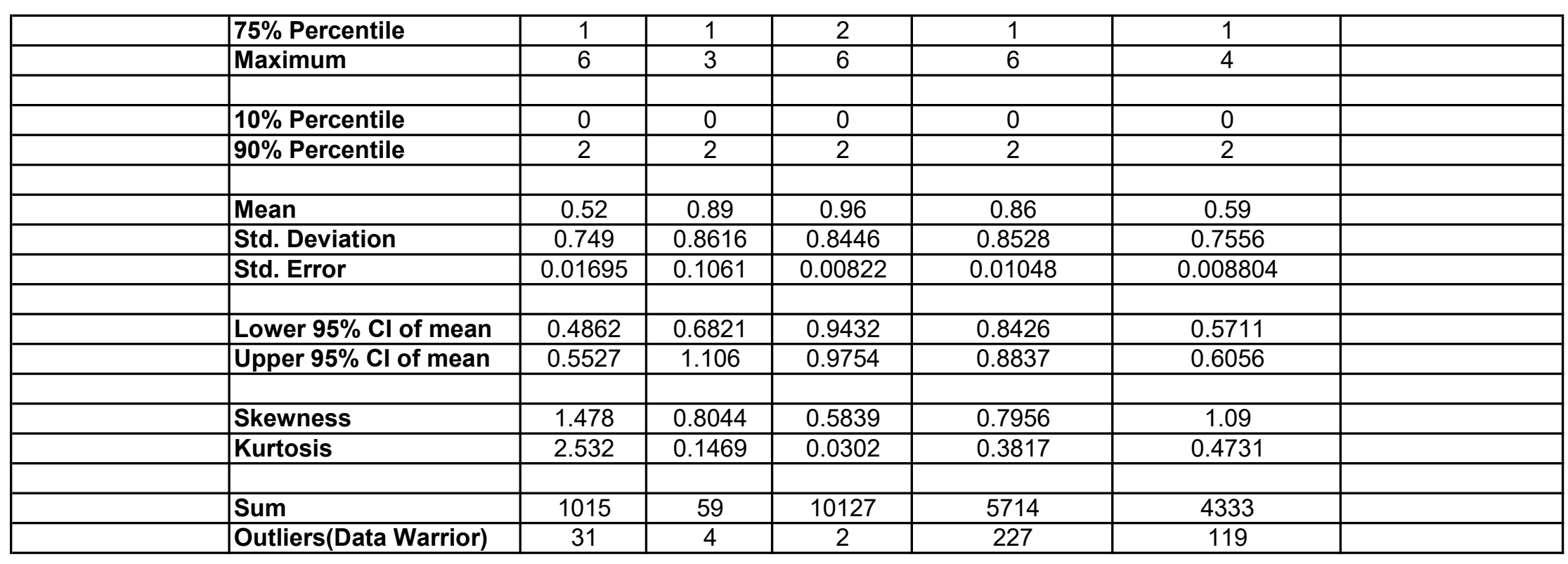




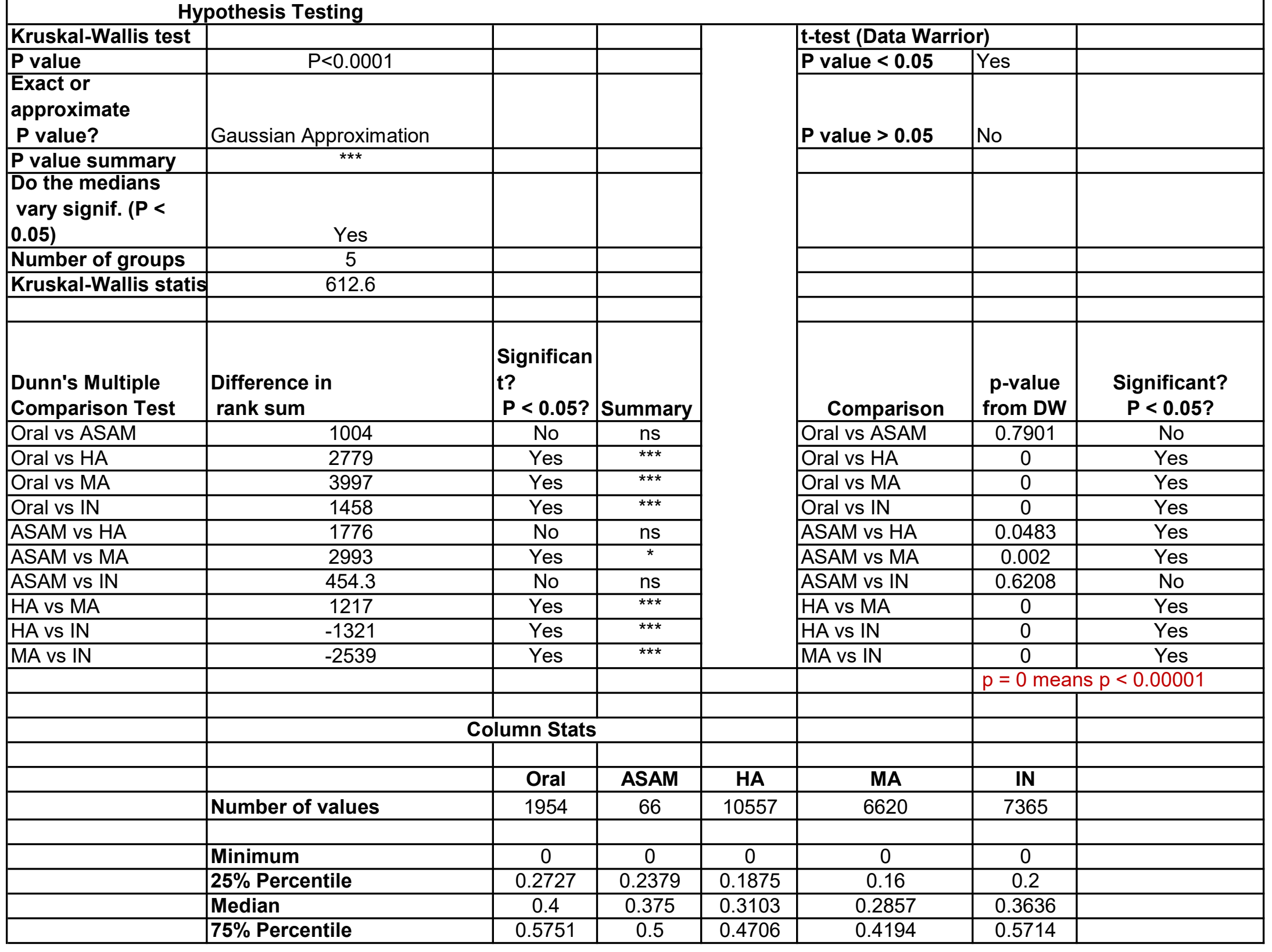




\begin{tabular}{|l|l|c|c|c|c|c|c|}
\hline & Maximum & 1 & 1 & 1 & 1 & 1 & \\
\hline & & & & & & & \\
\hline & 10\% Percentile & 0.13 & 0.11 & 0.09 & 0.07 & 0.07 & \\
\hline & 90\% Percentile & 0.78 & 0.95 & 0.71 & 0.59 & 0.87 & \\
\hline & & & & & & & \\
\hline & Mean & 0.4329 & 0.4237 & 0.3552 & 0.314 & 0.4067 & \\
\hline & Std. Deviation & 0.2373 & 0.2756 & 0.2356 & 0.2123 & 0.277 & \\
\hline & Std. Error & 0.005368 & 0.03393 & 0.00229 & 0.00261 & 0.003228 & \\
\hline & & & & & & & \\
\hline & Lower 95\% Cl of mean & 0.4223 & 0.3559 & 0.3508 & 0.3089 & 0.4004 & \\
\hline & Upper 95\% Cl of mean & 0.4434 & 0.4914 & 0.3597 & 0.3191 & 0.4131 & \\
\hline & & & & & & & \\
\hline & Skewness & 0.4463 & 0.89 & 0.9054 & 0.9989 & 0.5906 & \\
\hline & Kurtosis & -0.2561 & -0.2012 & 0.3799 & 1.041 & -0.5006 & \\
\hline & & & & & & & \\
\hline & Sum & 845.8 & 27.96 & 3750 & 2079 & 2996 & \\
\hline
\end{tabular}




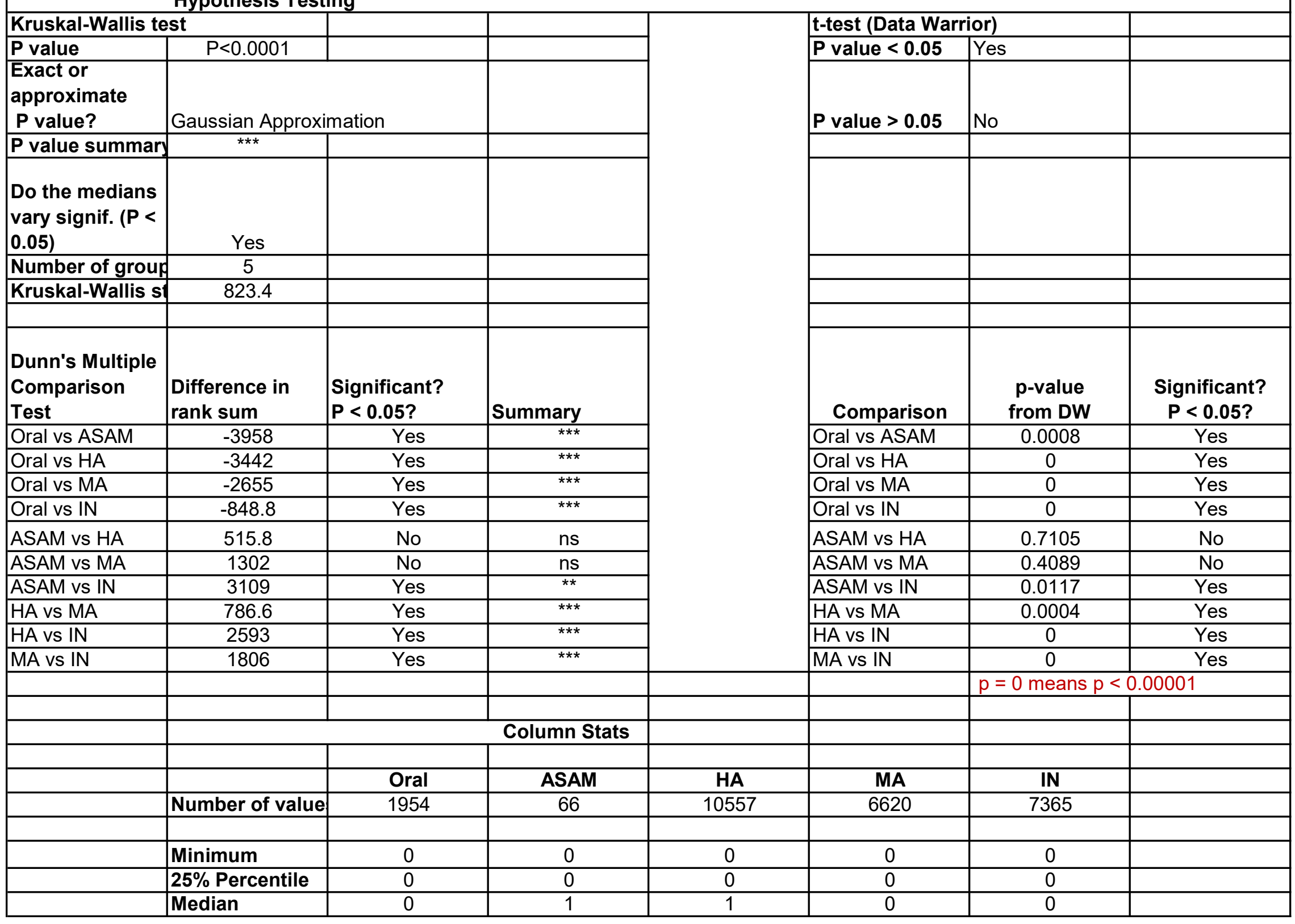




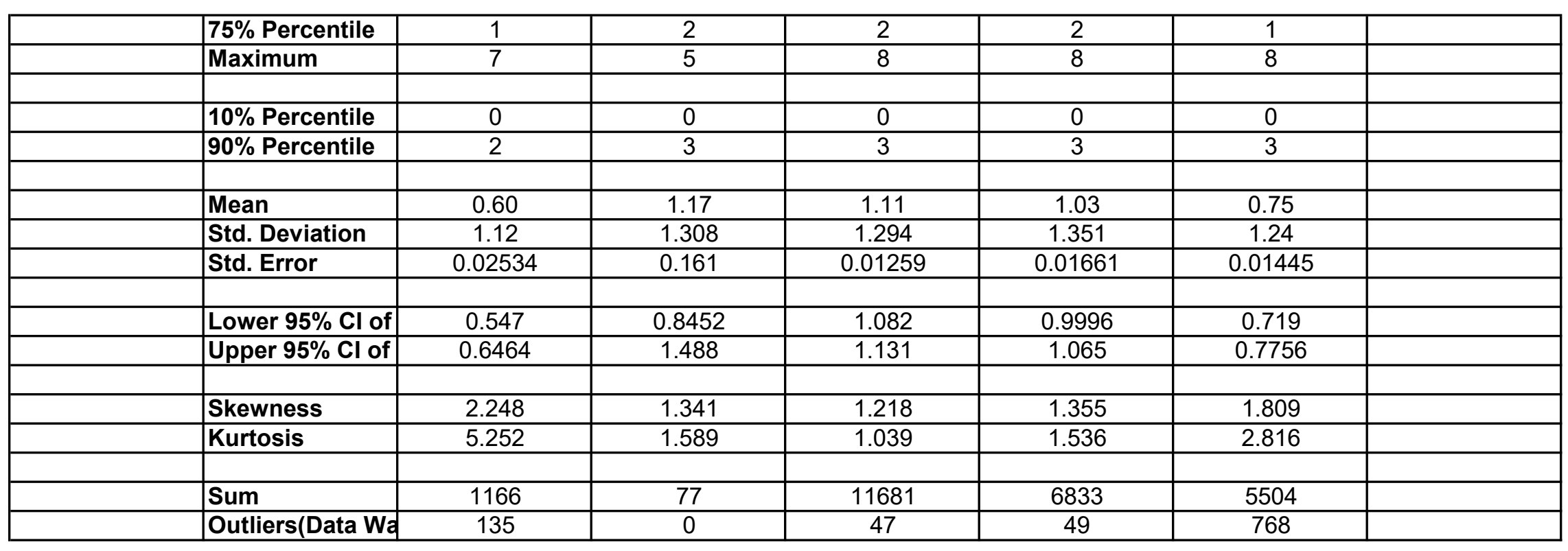




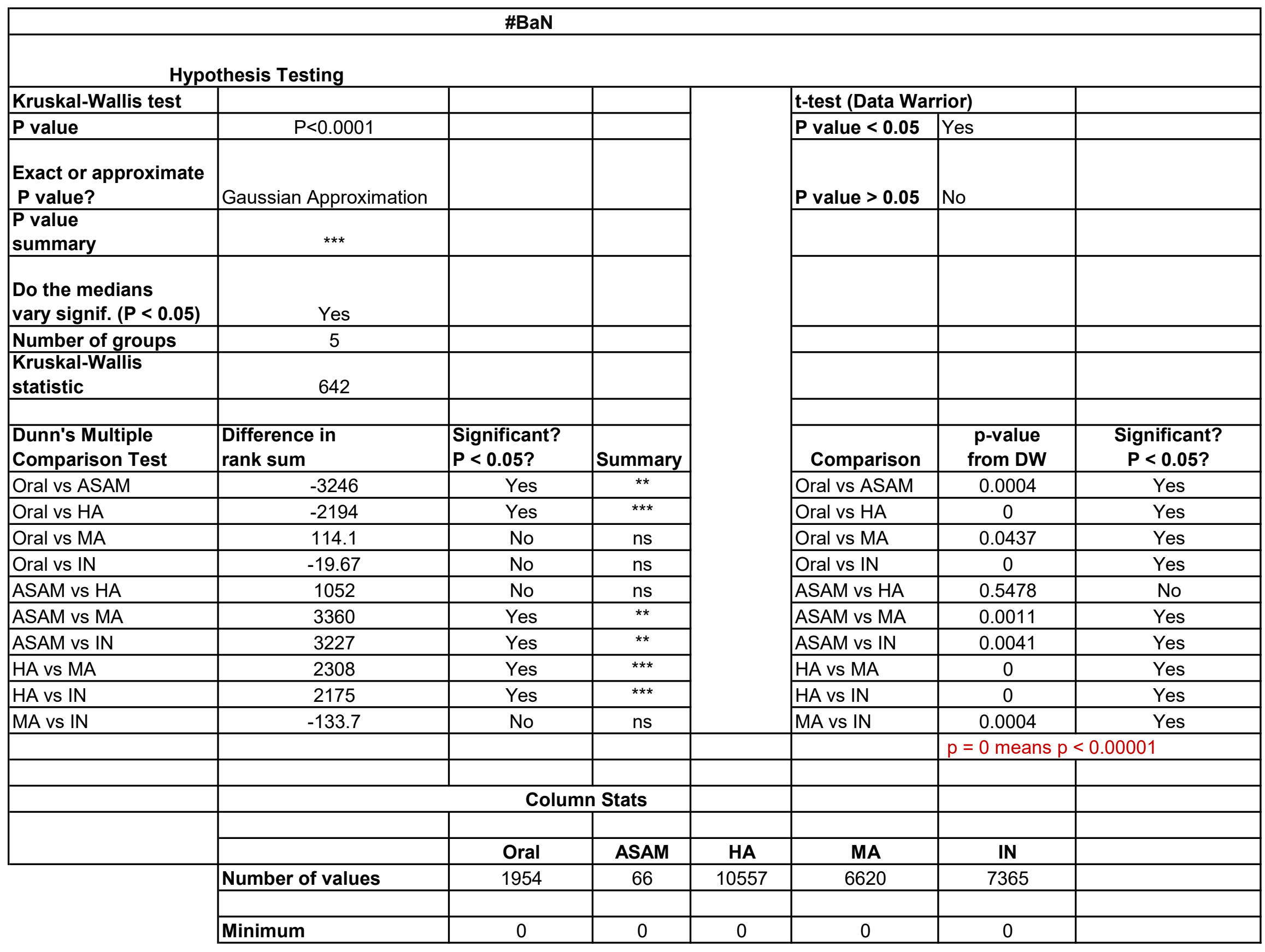




\begin{tabular}{|c|c|c|c|c|c|c|}
\hline 25\% Percentile & 0 & 0 & 0 & 0 & 0 & \\
\hline Median & 1 & 1 & 1 & 0 & 0 & \\
\hline 75\% Percentile & 1 & 2 & 2 & 1 & 1 & \\
\hline Maximum & 5 & 5 & 6 & 6 & 6 & \\
\hline $10 \%$ Percentile & 0 & 0 & 0 & 0 & 0 & \\
\hline $90 \%$ Percentile & 1 & 2 & 2 & 2 & 2 & \\
\hline Mean & 0.62 & 1.09 & 1.02 & 0.66 & 0.72 & \\
\hline Std. Deviation & 0.7248 & 1.019 & 1.105 & 0.8806 & 0.9911 & \\
\hline Std. Error & 0.0164 & 0.1254 & 0.01076 & 0.01082 & 0.01155 & \\
\hline Lower $95 \% \mathrm{Cl}$ of mean & 0.5886 & 0.8405 & 0.9938 & 0.6392 & 0.6939 & \\
\hline Upper $95 \% \mathrm{Cl}$ of mean & 0.6529 & 1.341 & 1.036 & 0.6816 & 0.7391 & \\
\hline Skewness & 1.331 & 1.254 & 1.016 & 1.495 & 1.447 & \\
\hline Kurtosis & 3.221 & 2.657 & 0.6973 & 2.801 & 1.887 & \\
\hline Sum & 1213 & 72 & 10714 & 4372 & 5277 & \\
\hline Outliers(Data Warrior) & 25 & 0 & 12 & 230 & 451 & \\
\hline
\end{tabular}

